\title{
Der Gang in den Schatten der Macht
}

\section{Warum engagiert man sich überhaupt in einer Klein(st)partei?}

\author{
Jan-Eric Bartels $\mathbb{D}$ - Frederik Springer
}

Eingegangen: 29. September 2020 / Überarbeitet: 3. Mai 2021 / Angenommen: 10. Mai 2021 / Online publiziert: 29. Juni 2021

(C) Der/die Autor(en) 2021

Zusammenfassung Ein erheblicher Anteil der Parteimitglieder in Deutschland entfällt auf Klein(st)parteien. So stellt die Gruppe der Klein(st)parteien summiert die drittmeisten Parteimitglieder in Deutschland. In Anbetracht einer wachsenden Anzahl und eines zunehmenden Stimmenanteils von Klein(st)parteien ist von einer steigenden Relevanz ihrer Mitgliedschaft auszugehen. Über diese große Gruppe ist, trotz vieler Untersuchungen von Parteimitgliedern im Allgemeinen, bisher sehr wenig bekannt. Dieser Beitrag ermittelt mit Schwerpunkt auf dem General-IncentivesModell die Motivationen für den „Gang in den Schatten der Macht“. Mittels Rückgriff auf Daten der Deutschen Parteimitgliederstudie 2009 wird gezeigt, dass die Mitgliedschaft in einer Klein(st)partei vor allem durch die Unzufriedenheit mit politischen Akteuren und dem System insgesamt sowie aus ideologischer Motivation gespeist wird. Klein(st)parteimitglieder sind ferner aktiver in ihrer Partei, verknüpfen mit ihrer Mitgliedschaft eher die Übernahme von Parteiämtern und ziehen eine größere Befriedigung aus ihrer Mitgliedschaft.

Schlüsselwörter Parteimitglieder $\cdot$ Kleinparteien · Kleinstparteien · GeneralIncentives-Modell $\cdot$ Motivationen

\footnotetext{
Jan-Eric Bartels $(\bowtie)$

Institut für Politikwissenschaft, Georg-August-Universität Göttingen, Platz der Göttinger Sieben 3, 37073 Göttingen, Deutschland

E-Mail: jan-eric.bartels@uni-goettingen.de

Frederik Springer

Institut für Politikwissenschaft, Leibniz Universität Hannover, Schneiderberg 50, 30167 Hannover, Deutschland

E-Mail: f.springer@ipw.uni-hannover.de
} 


\title{
Walking in the Shadows of Power
}

Why Get Involved in a Small or Marginal Party at all?

\begin{abstract}
A considerable proportion of party members in Germany belong to small and marginal parties. Thus, the group of small and marginal parties is in third place when it comes to party members in Germany. In view of the growing number and share of votes of small and marginal parties, it can be assumed that their membership is becoming increasingly relevant. Little is known about this large group, despite many studies centering on party members in general. This paper focuses on the general incentives model to determine the motivations for "going into the shadow of power." Using data from the German Party Membership Study of 2009, it is shown that membership in a small or marginal party is mainly driven by dissatisfaction with political actors and the political system and by ideological motivation. Members of small and marginal parties are also more active in their party, tend to associate their membership with the assumption of a party office, and derive greater satisfaction from their membership.
\end{abstract}

Keywords Party members $\cdot$ Small parties $\cdot$ Marginal parties $\cdot$ General incentives model $\cdot$ Motivations

\section{Einleitung}

Über die Motivation für die Mitgliedschaft in einer Partei ist bereits einiges bekannt. So spielen dort sozialpsychologische Aspekte, wie auch generelle Nutzenerwägungen eine Rolle (Hoffmann 2011; Seyd und Whiteley 1992; Koo 2021; Gomez et al. 2019; Achury et al. 2020). Diesen Untersuchungen ist jedoch gemein, dass sie in der Regel durch die Betrachtung der etablierten Parteien oder der Parteimitgliedschaft im Aggregat nur einen Ausschnitt der Parteienlandschaft und seiner Mitglieder abbilden oder sehr unterschiedliche Parteien in einer Gesamtschau betrachten.

So gibt es abseits dieser etablierten Parteien noch weitere Parteien, die im sogenannten „Schatten der Macht“ (Rowold 1974) agieren. Diese Klein- und Kleinstparteien sind allerdings ein selten untersuchtes Feld. Schlaglichtartig betrachtet wurden der Effekt von kleineren Parteien auf die Wahlergebnisse der großen Parteien (Chamberlain 2012; Luks et al. 2003), die Gründe für die Wahl von kleineren Parteien (Borisyuk et al. 2007; Bélanger 2004; Allen und Brox 2005), Organisationsanforderungen (van den Boom 1999) sowie ihre Einordnung im Parteiensystem (Rowold 1974). Doch systematische Untersuchungen darüber, welche Gründe für eine Parteimitgliedschaft bei diesen Parteien vorliegen, sind abseits von Einzelfallstudien (Gomez et al. 2019; Whiteley et al. 2019) bisher nicht durchgeführt worden. Für ein nuanciertes Bild von Parteimitgliedschaft ist allerdings eine Analyse der Stärke der unterschiedlichen Anreize für bestimmte Parteitypen unerlässlich. Da die Mitgliedschaft in einer Partei zu den wenigen kostenintensiven Beteiligungsformen mit nennenswerter Verbreitung gehört, trägt ihre ganzheitliche Betrachtung auch maßgeblich zum Verständnis politischer Partizipation der Bürger in ihrer Gesamtheit bei. In Anbetracht der zunehmenden Anzahl von Klein(st)parteien liefert die Betrachtung 
der Motivation des Beitritts einer Klein(st)partei zudem einen ersten Anhaltspunkt dazu, warum solche Parteien einen größeren Zulauf verzeichnen. ${ }^{1}$

Klein(st)parteien zeichnen sich dadurch aus, dass sie insbesondere auf Bundesebene nicht vertreten sind. ${ }^{2}$ Es sind Parteien, die bei Wahlen in der Regel nur unter der Kategorie „Sonstige“ verbucht werden. Sie tauchen kaum in der Medienlandschaft auf und verfügen über wenig Macht, da sie, wenn überhaupt, selten in Parlamenten sitzen und einen geringeren Organisationsgrad aufweisen als die großen etablierten Parteien. Dennoch engagiert sich eine beträchtliche Zahl der Bürger in solchen kleinen Parteien. Diese Arbeit versucht, die motivationalen Aspekte für die Mitgliedschaft in Klein(st)parteien zu ergründen und verbindet das General-Incentives-Modell (GIM) mit Motivationsmerkmalen, die sich aus der bisherigen Forschung zu Klein(st)parteien ableiten lassen.

In Anbetracht der fehlenden Machtperspektive stellt sich unweigerlich die Frage, warum diese kleinen Parteien überhaupt gegründet werden und vor allem, warum sie teilweise auch über einen längeren Zeitraum überleben. Die Ergründung der Beitrittsmotive ihrer Mitglieder trägt wesentlich zum Verständnis dieser grundlegenden Fragen bei. Die postalische Befragung der Deutschen Parteimitgliederstudie 2009 (Spier et al. 2011) zeigt für die Mitglieder der Parlamentsparteien, dass diese vor allem durch 1) kollektive, politische, 2) altruistische sowie 3) expressive Anreize zum Parteibeitritt motiviert wurden. Bei Klein(st)parteien ist jedoch vor allem in Bezug auf den erstgenannten Anreiz fraglich, inwieweit diese motivationalen Aspekte zutreffend sein können. Da die Partei kaum Ressourcen zur Verfügung hat und auch kaum in den Medien präsent ist, ist es schwerlich möglich, die sowieso kaum vorhandenen Einflussmöglichkeiten dieser Partei zu steigern. Vielmehr sind für Mitglieder von Kleinstparteien eher selektive, prozessbezogene sowie ideologische Anreize zu erwarten. Erstere ergeben sich aus dem Spaß an der politischen Arbeit, abzüglich der Unstimmigkeiten, die in großen Parteien auftreten können. Letztere ergeben sich aus ideologischen Positionen, deren Durchsetzung sich in einer großen Partei kaum realisieren lassen.

Während die soeben referenzierte Analyse ausschließlich auf Stichproben der Parteimitgliedschaften der etablierten Parteien beruht, lässt die ebenfalls im Rahmen der Deutschen Parteimitgliederstudie 2009 durchgeführte Telefonbefragung zusätzlich die Untersuchung von Mitgliedern der normalerweise unter „Sonstiges“ gezählten Parteien zu.

Es zeigt sich, dass Klein(st)parteimitglieder eine verminderte Unterstützung für das politische System und die darin agierenden politischen Akteure aufweisen. Mit ihrer eigenen Mitgliedschaft sind sie hingegen überproportional zufrieden. Innerhalb ihrer jeweiligen Partei weisen sie im Allgemeinen eine hohe Aktivität auf und haben entsprechend eher als Mitglieder von etablierten Parteien die Erwartung, Parteiämter

\footnotetext{
1 Dies ist insbesondere von Interesse, da Kselman et al. (2016) bereits darlegen konnten, dass eine höhere effektive Anzahl an Parteien im System zu mehr (erfolgreichen) neuen Parteien führt. Im Hinblick auf die anscheinende Etablierung der AfD ist es dementsprechend möglich, dass künftig weitere Klein(st)parteien beziehungsweise neue Parteien beachtliche Erfolge erzielen können.

2 Eine Definition des Konzepts der Klein(st)partei wird folgend im Abschnitt ,2.1. Parteimitglieder in etablierten Parteien und Klein(st)parteien“ vorgenommen.
} 
zu übernehmen. Wie Mitglieder großer Parteien sehen sie ihre Mitgliedschaft als eigenen Beitrag zum Funktionieren der Demokratie. Ihre Parteimitgliedschaft ist außerdem überproportional häufig ideologisch motiviert.

Dieser Beitrag schließt hiermit vor allem an zwei Debatten an. Erstens erweitert dieser Beitrag die Debatte zur Motivation von Parteimitgliedern. Zum einen zeigt sich, dass es Motivationsfaktoren gibt, die klar über alle Parteigrößen hinweg Geltungskraft haben. Insbesondere die zu den erklärungsmächtigen Motivationsfaktoren der Partiemitgliedschaft gehörenden altruistischen Anreize (Gomez et al. 2019; Hoffmann 2011) sind in gleichem Ausmaß bei Klein(st)parteimitgliedern sowie bei Mitgliedern großer Parteien zu finden. Zum anderen gibt es jedoch auch Motivationsfaktoren, die nach Parteigröße eine unterschiedliche Bedeutung für die Parteimitgliedschaft aufweisen. Es stellte sich vor allem heraus, dass nicht ausschließlich die Zufriedenheit mit dem demokratischen System als Motivator für die Mitgliedschaft wirken kann (Schäfer 2013; Anderson 1998), sondern - im Fall von Klein(st)parteien - eine Mitgliedschaft sogar durch Unzufriedenheit begünstigt wird. Generell gilt überdies, dass das GIM auch bei Klein(st)parteien die Parteimitgliedschaft zu erklären vermag. Diese Anreize sind dort jedoch anders gewichtet als bei den etablierten Parteien. Zweitens schließt dieser Beitrag an die Forschung zu Klein(st)parteien an. So zeigt sich, dass diese Parteien nicht nur Unzufriedene an der Wahlurne anziehen (Wuttke 2020; Allen und Brox 2005), sondern auch die Mitgliedschaft zu einem größeren Teil aus tendenziell Unzufriedenen besteht. Vorhergehende Untersuchungen neuer Parteien deuten darauf hin, dass eine programmatisch starke Unterscheidbarkeit für das Überleben dieser Parteien wichtig sei (Bolleyer 2013). Mit Hinblick auf die Mitgliedschaft lässt sich diese programmatische Unterscheidbarkeit ebenfalls erklären. Die Mitglieder von Klein(st)parteien sind nämlich zumeist radikaler eingestellt als es die Mitglieder etablierter Parteien sind.

Unsere Ausführungen beginnen mit der Erläuterung der theoretischen Grundlagen. Diese umfasst eine Definition der Begrifflichkeiten der Klein- und Kleinstparteien sowie eine Darlegung der zunehmenden Relevanz dieses Parteityps. Der Abschnitt beinhaltet darüber hinaus eine Beschreibung des General-Incentives-Modells und der Funktionen von Klein(st)parteien. Aus dem Anreizmodell und den spezifischen Parteifunktionen ergibt sich sodann auch die Herleitung unserer Hypothesen. Im Anschluss an die Beschreibung der verwendeten Datenbasis, der Telefonbefragung der Deutschen Parteimitgliederstudie 2009, erfolgt die Überprüfung der Hypothesen anhand von Mittelwertvergleichen. Unsere Abhandlung schließt mit einem Fazit, das die Ergebnisse zusammenfasst und Forschungsdesiderata aufzeigt.

\section{Theorie}

\subsection{Parteimitglieder in etablierten Parteien und Klein(st)parteien}

Grundsätzlich zeigt sich, dass vor allem ressourcenstarke Gruppen in Parteien engagiert sind (Spier 2019, S. 298). Zumeist verfügen Parteimitglieder über einen höheren Bildungsabschluss und befinden sich im letzten Drittel ihres Berufslebens (Achury et al. 2020, S. 57). Obwohl es sich dabei um wichtige Bestimmungsfaktoren 
Tab. 1 Anreize für den Parteieintritt

\begin{tabular}{ll}
\hline Ideologische Anreize (H2) & $\begin{array}{l}\text { Unterstützung bestimmter ideologischer Ideale } \\
\text { Kollektive politische Anreize (H2) }\end{array}$ \\
$\begin{array}{l}\text { Altruistische Anreize (H3) } \\
\text { Selektive, prozessbezogene Anreize } \\
\text { (H4) }\end{array}$ & $\begin{array}{l}\text { Beitrag zum Funktionieren der Demokratie leisten } \\
\text { Persönlicher Vorteil entsteht aus Mitarbeit in Partei }\end{array}$ \\
$\begin{array}{l}\text { Selektive, ergebnisbezogene Anreize } \\
\text { (H4) }\end{array}$ & Persönlicher Vorteil entsteht als Ergebnis der Mitgliedschaft \\
$\begin{array}{l}\text { Normative Anreize (H4) } \\
\text { Expressive Anreize (H4) }\end{array}$ & Erfüllung bestimmter Erwartungen des Umfelds \\
\hline
\end{tabular}

Die Kurzbeschreibungen der Anreiztypen sind Laux (2011, S. 63) entnommen

handelt (Hoffmann 2011, S. 82), können sie nicht die dem Beitritt zugrundeliegende Motivation erklären. Es handelt sich dabei letztlich um die Kontextfaktoren, die einen Beitritt begünstigen.

Eine etablierte Kategorisierung motivationaler Faktoren einer Parteimitgliedschaft ist das sogenannte General-Incentives-Modell (Seyd und Whiteley 1992; Hoffmann 2011; Gomez et al. 2019). Das Modell basiert auf der Rational-Choice-Theorie, hat diese allerdings um weitere motivationale Faktoren abseits von materiellen Kosten und Nutzen erweitert. Es umfasst sieben positive und drei negative Anreize ${ }^{3}$ (siehe Tab. 1 für die positiven Anreize), auf deren Grundlage Individuen eine etwaige Parteimitgliedschaft beurteilen (Laux 2011). Bisherige Untersuchungen der Parteimitglieder in Deutschland zeigen auf, dass die Mitgliedschaft vor allem durch drei dieser motivationalen Faktoren bestimmt ist. 1) Personen wollen sich für die Ziele der Partei einsetzen und deren Einfluss steigern. Diese kollektiven, politischen Anrei$z e$ sind letztlich auch ein wichtiger Faktor für die Aktivität in der Partei (Spier 2019). 2) Altruistische Anreize drücken den Willen aus, einen Beitrag zum Funktionieren der Demokratie zu leisten, und dabei insbesondere die Motivation, durch die Parteimitgliedschaft seiner „Verantwortung als Bürger“ nachzukommen. 3) Expressive Anreize stellen schließlich die Bekundung der Unterstützung der Partei und ihrer Politiker dar. Dementsprechend soll der Sympathie für die Partei oder ihrer Persönlichkeiten an der Parteispitze Ausdruck verliehen werden (Hoffmann 2011). Diese Anreize weisen auch in anderen Ländern eine hohe Erklärungskraft auf (Gomez et al. 2019; Poletti et al. 2019). Letztlich entscheiden in Deutschland auch Kosten in großem Maße darüber, ob man sich für eine Parteimitgliedschaft entscheidet oder nicht (Hoffmann 2011). In anderen Ländern lässt sich dies zum Teil nicht in der Deutlichkeit feststellen (Gomez et al. 2019).

Studien, die sich mit Parteimitgliedern beschäftigen, sind in der Regel auf die Parlamentsparteien fokussiert (Laux 2011) oder betrachten Parteimitgliedschaft auf einem aggregierten Level (Giugni und Grasso 2021; Hoffmann 2011). Zwar gibt es

\footnotetext{
3 Die negativen Anreize, also Mitgliedschaftsbeiträge, Opportunitätskosten und Arbeitsleid, sind jedoch für Klein(st)parteien genauso zu erwarten wie für die etablierten Parteien und dienen somit nicht der Unterscheidbarkeit. Gomez et al. (2019) zeigten hierzu bereits auf, dass bei der Aktivität der Parteimitglieder die Kosten keinen bedeutenden Faktor dargestellt haben, die zur Unterscheidung von Vollmitgliedern und Sympathisanten beitragen würden.
} 
durchaus auch Arbeiten, die sich mit den kleineren Parteien des Systems beschäftigen, jedoch geschieht dies in der Regel in Form von Einzelfallstudien (Whiteley et al. 2019; Biehl und Kranenpohl 2011) oder beschränkt auf bestimmte politische Strömungen (Gomez et al. 2019).

Klein- und Kleinstparteien werden in der Literatur unterschiedlich definiert und auch für die Operationalisierung des Konzepts werden variierende Kennzahlen herangezogen. So gibt es zum einen den Ansatz, Parteien anhand ihres Einflusses zu kategorisieren ${ }^{4}$ (van den Boom 1999; Nestler 2013). Zum anderen erfolgt die Einordnung nach dem Wahlerfolg (Kranenpohl und Niedermayer 2013; Hirano und Snyder 2007; Lee 2014; Raymond und Wessel Tromborg 2016). Dies geschieht wiederum entweder auf Grundlage des Stimmenanteils oder anhand der Sitze im Parlament. Hier schließen wir uns der Einordnung anhand der Mandate im Parlament an, da dies, im Gegensatz zu Kriterien wie dem Ausmaß des politischen Einflusses, eine eindeutige Kategorisierung ohne subjektiven Ermessungsspielraum ermöglicht. Da der politische Einfluss allerdings durch parlamentarische Repräsentanz maßgeblich bedingt sein sollte, geht auch mit unserer Operationalisierung einher, dass Klein(st)parteien im Allgemeinen maximal über geringe politische Gestaltungsmöglichkeiten verfügen. Dementsprechend ist nach unserer Konzeptualisierung eine Klein(st)partei eine Partei, die entweder über keine Sitze im Parlament verfügt, oder zwar über Sitze verfügt, jedoch nicht auf allen Ebenen - insbesondere nicht auf Bundesebene. Hiermit verbleibt die Definition im Kontext der bisherigen Ansätze, vereint hauptsächlich die Unterteilungen und schafft eine sprachliche Klarheit. Einen Vergleich der Kriterien zur Einordnung liefert Tab. 8 im Online-Anhang.

Mitunter fallen Klein(st)parteien ebenso in die Kategorie der Nischenparteien. Allerdings ist dieses Konzept in Bezug auf die Größe der Partei sowie deren Wirkmächtigkeit uneindeutiger. In der Regel beziehen sich diese Definitionen auf Parteien, die parlamentarische Repräsentation (insbesondere auch auf Bundesebene) erfahren oder zumindest in die Nähe ebenjener kommen, jedoch hauptsächlich ein eingeschränktes und von den anderen Parteien des Systems stark abgegrenztes programmatisches Profil aufweisen (Meyer und Miller 2015; Meguid 2005). Durch die Einteilung anhand der Programmatik wären letztlich relativ große, jedoch programmatisch besondere, Parteien in einem Sample mit kleinen und vergleichsweise erfolgloseren Parteien zusammengefasst. Im Interesse dieser Arbeit liegt jedoch das Engagement in einer Partei trotz eines unklaren unmittelbaren Nutzengewinns in Form der schlussendlichen Politikgestaltung.

Die Relevanz der Klein- und Kleinstparteien kann anhand von zwei Punkten deutlich veranschaulicht werden. Amorim Neto und Cox (1997) folgend wird die Effizienz der Reduzierung der Anzahl der Parteien in einem bestimmten System zum einen durch das Wahlsystem und seine Stärke beeinflusst. Dies bedeutet, dass „starke“ Systeme weniger Parteien hervorbringen. Zum anderen prägt auch die Anzahl der in einem Land vorherrschenden Cleavages die Anzahl der Parteien. Man ging davon aus, dass Deutschland über ein „starkes“ Wahlsystem verfügt, was auf die Dominanz der beiden großen Parteien vor allem in der Mitte der 1970er- bis

\footnotetext{
4 Die Einteilung anhand des Einflusses bei van den Boom (1999) ist im Grunde angelehnt an die Einlassungen Sartoris (1976) zu relevanten Parteien des Parteiensystems.
} 


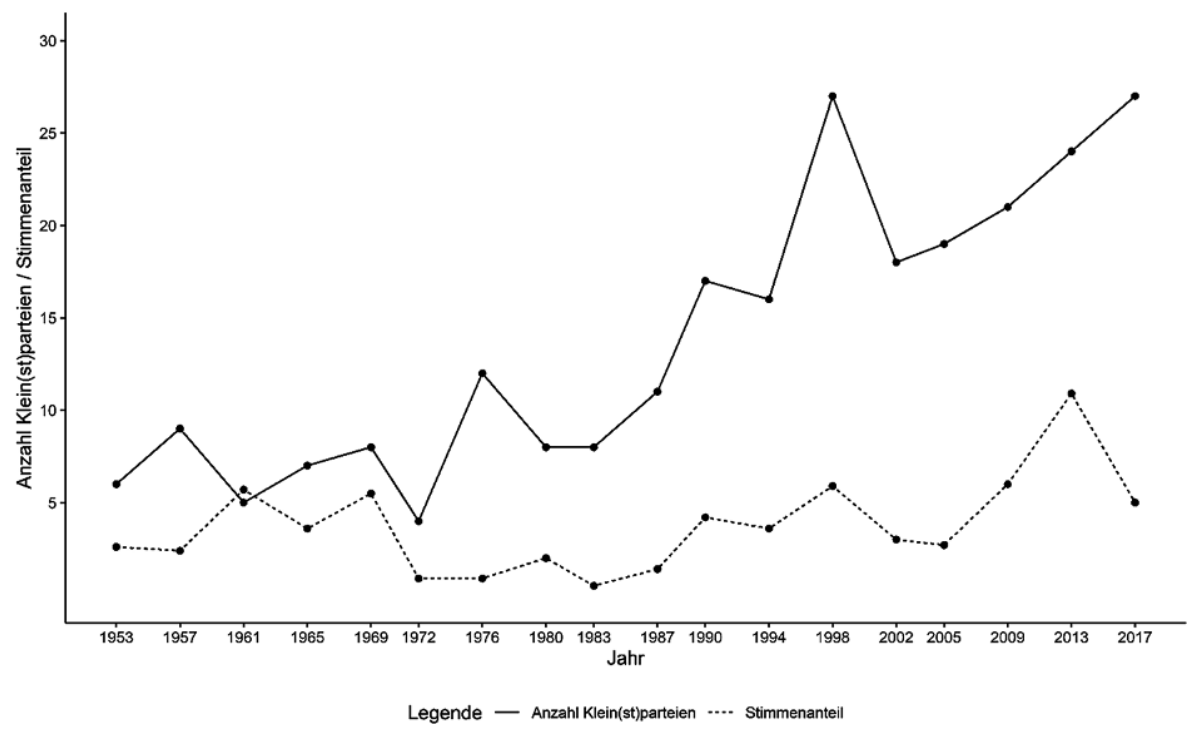

Abb. 1 Anzahl (absolut) und Stimmen (in \%) der Klein(st)parteien bei Bundestagswahlen seit 1953

1980er-Jahre und die 5\%-Hürde für den Einzug ins Parlament zurückzuführen ist. Seitdem haben sich weder die Cleavages (Magin et al. 2009) noch das Wahlsystem (Behnke 2007; Hartmann 2013) stark verändert. Trotz dieser Umstände sehen wir einen massiven Anstieg der Zahl von Klein(st)parteien, die an den Bundestagswahlen teilnehmen (Abb. 1), und sogar ihr Stimmenanteil hat seit den 1970er-Jahren zugenommen. ${ }^{5}$ Eine mögliche Erklärung könnte die zunehmende Individualisierung sein (Dalton 2000).

Natürlich haben die Klein- und Kleinstparteien längst nicht so viele Mitglieder wie die Bundestagsparteien. Doch ihre Anzahl ist höher als zunächst vermutet. Zwar verfügen derartige Parteien jeweils über relativ wenig Mitglieder, aufsummiert jedoch stellen sie die viertgrößte Gruppe dar (Tab. 2). ${ }^{6}$ Diese Berechnung beruht auf den Mitgliedszahlen der Klein(st)parteien, die bei der Bundestagswahl 2017 oder der Europawahl 2019 antraten. Wir berücksichtigen in dieser Auflistung also solche Parteien nicht, die bisher nicht genügend Unterschriften für eine Wahlteilnahme sammeln konnten. Auch dürfte die Dunkelziffer etwas höher sein, da die Mitgliedszahlen von Kleinstparteien deutlich schlechter zugänglich sind als die von etablierten Parlamentsparteien, und es teilweise nicht ersichtlich ist, wie aktuell ihre Mitgliederkarteien noch sind.

\footnotetext{
${ }^{5}$ Die Spitze des Stimmenanteils 2013 lässt sich durch das Aufkommen der AfD erklären, die folgend jedoch mit dem Einzug in den Bundestag aus der Kategorisierung herausfällt.

${ }^{6}$ Wenngleich die Klein(st)parteienlandschaft ideologisch durchaus heterogen ist, erscheint diese Zusammenlegung dennoch sinnvoll. Diese Parteien eint letztlich, dass sie keine parlamentarische Repräsentation (vor allem auf Bundesebene) erlangt haben und dementsprechend über äußerst wenig Ressourcen verfügen. Hierdurch ist auch die motivierende Fragestellung begründet. Es ist beispielsweise nicht klar, warum einige Menschen in grünen Klein(st)parteien aktiv werden, wo mit Bündnis 90/Die Grünen eine Partei des gleichen Themenfeldes bereits parlamentarische Repräsentation erhielt.
} 
Tab. 2 (Aufsummierte) Mitgliederzahlen der bei der Bundestagswahl 2017 oder Europawahl 2019 angetretenen etablierten Parteien und Klein(st)parteien

\begin{tabular}{lllllllll}
\hline Partei & SPD & CDU & CSU & Klein(st)parteien & GRÜNE & FDP & $\begin{array}{l}\text { Die } \\
\text { Linke }\end{array}$ & AfD \\
Mitglieder & 437.454 & 414.905 & 138.354 & 83.266 & 75.311 & 63.912 & 62.016 & 33.516 \\
\hline
\end{tabular}

Grundsätzlich zeigt sich, dass ein großer Teil der bei der Bundestagswahl 2017 oder Europawahl 2019 angetretenen Klein(st)parteien eher neuere Parteien sind (siehe Tab. 3). Allerdings ist dies mitnichten ausschließlich der Fall. So traten zu diesen Wahlen auch zahlreiche Klein(st)parteien an, die bereits seit längerer Zeit existieren. Unter diesen älteren Klein(st)parteien befinden sich zudem einige Parteien, die mitunter auch etwas größere Höhenflüge verzeichnen (NPD, ÖDP), aber auch solche, die noch gar keine großen Erfolge verbuchen konnten (SGP, MLPD).

Insgesamt können diese Parteien in 6 Cluster eingeordnet werden. Der erste Cluster ist jener der radikalen Rechten. Hierunter fällt als größter Vertreter beispielsweise die NPD. Sie ist mitunter auch eine der erfolgreicheren Klein(st)parteien, mit Einzügen in einige Landesparlamente und dem wiederholten Einzug ins Europaparlament. Zuletzt war sie vor allem aufgrund eines Verbotsverfahrens in den Schlagzeilen, in dessen Zuge zwar die Verfassungsfeindlichkeit festgestellt wurde, das jedoch aufgrund der geringen Wirkmächtigkeit und des desolaten Organisationsgrades der Partei keinen Erfolg hatte (Jesse 2018b). DIE RECHTE oder der III. Weg fallen ebenso in diese Kategorie, wobei erstere unter anderem durch die Aufstellung der bekannten und inhaftierten Holocaustleugnerin Ursula Haverbeck als Spitzenkandidatin für die Europawahlen 2019 aufgefallen ist (Speit 2020). Ein weiteres Cluster bilden die Umweltparteien. Hierunter sind zumeist Abspaltungen der GRÜNEN zu finden, wie ÖkoLinX, die unter anderem von Jutta Ditfurth gegründet wurde, oder die ÖDP, die maßgeblich von Herbert Gruhl gegründet wurde (Kranenpohl 2018). Die größte und erfolgreichste Partei ist hier die ÖDP, die bereits mehrfach ins europäische Parlament eingezogen ist und zudem eine der häufiger untersuchten Klein(st)parteien darstellt (Kranenpohl und Niedermayer 2013). Einen weiteren, etwas größeren Cluster bilden die esoterischen Parteien. Hier stechen vor allem Die Violetten hervor, die bereits seit Anfang der 2000er-Jahre existieren und dafür werben, spirituelle Aspekte im Sinne einer ,ganzheitlichen“ Realität stärker in die Politik einzubringen (Lewandowsky 2018).

Kommunistische Parteien bilden ein weiteres Cluster, mit der DKP als eine der prominenteren Vertreterinnen. Sie wurde vor allem aus der DDR unterstützt und war dementsprechend von der SED stark finanziell, aber auch organisatorisch abhängig. Seit der Wiedervereinigung spielt sie deshalb auch eine deutlich geringere Rolle in der Parteienlandschaft, auch weil sie sich zunehmend in Richtungsstreitigkeiten verliert (Jesse 2018a). Darüber hinaus gibt es zudem einige Parteien, die vor allem spezielle Bevölkerungsgruppen repräsentieren, wie Die Grauen, Familienpartei oder Die Frauen. Die Grauen und auch die Grauen Panther können dabei auf eine lange Tradition zurückblicken. Beide Parteien sind Abspaltungen der Partei Die Grauen Graue Panther, die bereits 1989 gegründet wurde, deren Bewegung allerdings schon in den 1970er-Jahren ihren Ursprung hatte und 2008 infolge eines Spendenskandals aufgelöst wurde (Schulze 2018). Daneben gibt es auch noch eine Reihe von Par- 


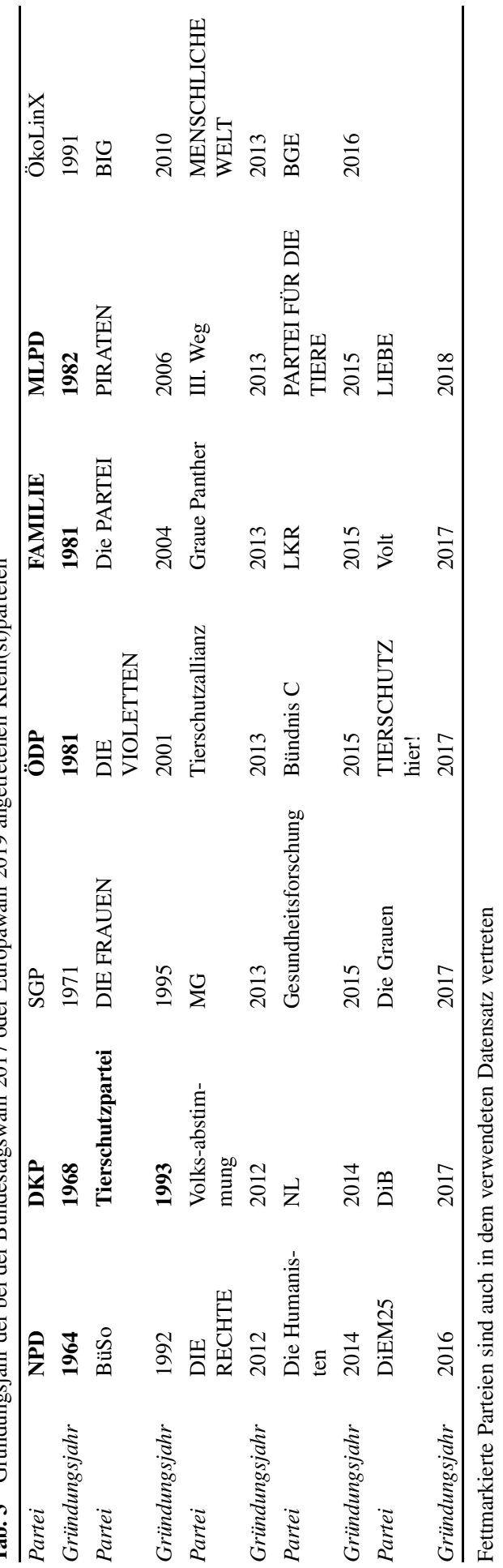


teien, die spezielle Partikularinteressen vertreten, wie Gesundheitsforschung oder die Magdeburger Gartenpartei. Diese beschäftigen sich zumeist mit Randthemen, wie beispielsweise der Bekämpfung altersbedingter Krankheiten im Falle der Partei Gesundheitsforschung (Küppers 2018) oder dem Erhalt von Kleingartenanlagen im Falle der Magdeburger Gartenpartei (Zimmer 2017).

Wir gehen davon aus, dass Mitglieder von Klein(st)parteien zumindest zum Teil andere Anreize haben als Mitglieder von etablierten Parteien. Dies ist vor allem durch die sehr begrenzten Macht- und Veränderungsmöglichkeiten von Klein(st)parteien begründet. Auch stehen Klein(st)parteien in der Regel nicht im Fokus der Öffentlichkeit, weshalb es beispielsweise noch eines weiteren Schrittes der eigenständigen Informationsbeschaffung bedarf, einer solchen Klein(st)partei beizutreten (oder sie gar zu gründen).

\subsection{Anreize bei Klein(st)parteien}

Klein(st)parteien standen bisher bei vergleichenden Mitgliederstudien nicht im Fokus. Dementsprechend ist auch über die Unterschiede der Motivation der Mitgliedschaft zwischen etablierten Parteien und Klein(st)parteien kaum etwas bekannt. Deshalb gibt es auch keinen klassischen Hypothesenkatalog für das Engagement in solchen Parteien, der für die vorliegende Analyse zugrunde gelegt werden könnte. Das GIM erklärt die Motivationen für den Parteibeitritt für die mindestens halbwegs relevanten Parteien im System relativ gut. Da Klein(st)parteien allerdings nicht genau die gleichen Funktionen erfüllen wie die etablierten Parteien, ist fraglich, inwieweit sich die motivationalen Faktoren auf diese Parteien beziehungsweise deren Mitglieder übertragen lassen. Welche Anreize des GIM für Klein(st)parteimitglieder besonders bedeutsam sind, leiten wir aus den bisherigen Ausführungen zu Klein(st)parteien ab.

Eine der bisher umfassenderen Analysen von Klein(st)parteien stammt von van den Boom (1999), welche unter anderem auch Interviews einiger Klein(st)parteivorsitzender beinhaltet. Van den Boom destilliert aus diesen Interviews Funktionen von Klein(st)parteien heraus, die letztlich jedoch vor allem die Motivationen des Beitritts beschreiben. Klein(st)parteien gelten demzufolge als 1) Auffangbecken der ewig Unzufriedenen, 2) Entwicklungsfeld neuer politischer Ideen, 3) Bewahrer politischer Traditionen, 4) Kontrolleure der Mächtigen und 5) „Ort der Vereinsmeierei“ (van den Boom 1999; Köhler 2006).

Unter anderem aufgrund der schwierigen empirischen Datenlage sind diese Motivationsanreize bisher kaum überprüft worden. Grundsätzlich liegt es jedoch im Bereich des Möglichen, zu testen, ob die Mitgliedermotivation diesen Einordnungen entspricht. Dementsprechend sollen die dargestellten Motivationsmöglichkeiten der Herleitung von Hypothesen über die Motivationen der Mitglieder von Klein(st)parteien dienen.

Mit Blick auf den ersten Motivationsfaktor - Auffangbecken der ewig Unzufriedenen - stellt van den Boom (1999, S. 264-265) fest, dass es zunächst immer Personen gibt, die sich durch die etablierten Parteien nicht vertreten fühlen. Bei diesen Personen gibt es teilweise ein sehr hohes Potenzial an politischem Idealismus. Die Möglichkeiten, die sich diesen Unzufriedenen schließlich bieten, sind begrenzt. Zum einen können sie resignieren und sich aus dem politischen Prozess verabschie- 
den. Zum anderen können sie eine Gruppe beziehungsweise einen Flügel innerhalb einer etablierten Partei gründen, um ihre Partikularinteressen dort mit Gleichgesinnten voranzubringen. Dies ist jedoch auch immer mit größeren Konflikten innerhalb einer Partei verbunden. Schließlich gibt es die Möglichkeit, eine eigene Partei zu gründen beziehungsweise einer Klein(st)partei beizutreten, die sich der eigenen Partikularinteressen annimmt. Der Motivationsgrund für diese Akteure sei, dass große Parteien oftmals eine monolithische Struktur sowie Machtkämpfe aufwiesen. Dies führe dazu, dass Idealismus in diesen Parteien weniger gefragt sei. Durch die Arbeit in einer eigenen Partei versprechen sich diese Akteure, sich stärker auf die eigene politische Arbeit konzentrieren zu können und sich nicht in parteitaktischen Manövern zu verlieren (van den Boom 1999, S. 264-267).

Diese Mitglieder sind dementsprechend unzufrieden mit dem System ${ }^{7}$ und insbesondere mit den etablierten Parteien. Mit Blick auf das Beispiel der ÖDP zeigt sich ebenfalls, dass deren Mitglieder die anderen Parteien in der Regel als eher schlecht einstufen (Kranenpohl 2008). Dies bedeutet allerdings nicht, dass Mitglieder von Klein(st)parteien zwangsläufig das politische System als solches ablehnen würden. Schließlich bleiben ihre Interessen nicht ohne Gehör, sondern werden lediglich ,ausgelagert“. Durch die Vertretung bestimmter Partikularinteressen tragen Klein(st)parteien somit letztlich zur Systemstabilität bei (van den Boom 1999, S. 266-267).

Die breitere Literatur unterstützt diese Ausführungen im Wesentlichen. So zeigt sich zunächst, dass Mitglieder großer Parteien grundsätzlich eine relativ hohe Zufriedenheit mit der Demokratie aufweisen (Vetter 2004; Höhne 2018). Hier gibt es jedoch auch Ausnahmen. So sind die Mitglieder von systemkritischeren Parteien wie Die LINKE und vor allem der AfD im Mittel deutlich unzufriedener (Höhne 2018). Im Bereich der Klein(st)parteien zeigte sich bei der kanadischen Reformpartei ein ähnliches Bild. Hier war der Hauptmotivator des Beitritts ebenfalls die Unzufriedenheit mit dem System und den bestehenden Parteien (Clarke et al. 2000). Dazu passt, dass auch Mitglieder der ÖDP in Deutschland das politische System als undurchlässiger betrachten (Biehl und Kranenpohl 2011). Am Beispiel der UKIP in Großbritannien konnte dagegen nicht festgestellt werden, dass die Aktivität in der Partei von der Unzufriedenheit mit der Demokratie abhängt (Whiteley et al. 2019). Allerdings wird in den Untersuchungen von Whiteley et al. (2019) die Aktivität in der Partei und nicht der Beitritt zu einer Partei behandelt. Entsprechend ändert dieser Befund nichts daran, dass wir insbesondere bei den Klein(st)parteien davon ausgehen können, dass die Unzufriedenheit mit dem System ein starker Anreiz für die Parteimitgliedschaft ist.

Daraus ergibt sich die erste Hypothese.

H1 Mitglieder von Klein(st)parteien sind unzufriedener mit dem System beziehungsweise der Demokratie als die Mitglieder von etablierten Parteien.

Grundsätzlich stellt die Ideologie einen großen Erklärungsfaktor für den Beitritt zu einer Partei dar (Heidar 1994, S. 75). Für kleine Parteien lässt sich sogar noch

\footnotetext{
7 Hier geht es dezidiert um das demokratische System als Ganzes. Unzufriedenheit über soziale Ungerechtigkeiten oder Unzufriedenheit mit anderen Policy-Feldern sind hierbei nicht gemeint.
} 
deutlicher feststellen, dass hier eine höhere Policy-Motivation vorherrscht als bei den größeren Pendants (Koo 2021, S. 198).

Diese Parteien entwickeln zuweilen auch neue politische Ideen. Einige warten sogar mit relativ langen Grundsatzprogrammen auf, manche haben detaillierte Programme zu bestimmten Politikfeldern. Diese Ideen lassen sich zwar teilweise auch in bestehende Schemata einordnen, doch ist die Positionierung bei diesen Klein(st)parteien letztlich eine andere als bei den etablierten Parteien (van den Boom 1999, S. 267-272).

Des Weiteren sorgen einige dieser Parteien dafür, dass alte Entwicklungslinien erhalten und historische Wurzeln betont werden. Dabei beleben sie teilweise den Wettbewerb durch Konzepte oder auch Organisationsformen vergangener Zeiten. Insbesondere alte politische Ideen finden sich in Klein(st)parteien oft wieder. Dies beinhaltet Ideen, die einst bereits umgesetzt oder zumindest im politischen Diskurs waren, aber mittlerweile herausgedrängt wurden. Als Beispiele können hierfür der Monarchismus sowie der Marxismus dienen, die sich in einigen Klein(st)parteien wiederfinden lassen (van den Boom 1999, S. 267-272).

Dies verdeutlicht, dass Klein(st)parteien nicht ausschließlich von mit dem System Unzufriedenen gespeist werden, sondern sich auch durch ideologisch motivierte Unterschiede von den größeren Parteien des Systems abheben. In den größeren Parteien gibt es durchaus viele ideologische „Misfits“ unter den - meist inaktiven - Parteimitgliedern (van Haute und Carty 2012, S. 893). Mitglieder von Klein(st)parteien haben vermutlich eher von ihrer „Exit Option“ (Hirschman 1970) Gebrauch gemacht oder sich aufgrund der ideologischen Differenzen gar nicht erst für eine der größeren Parteien entschieden. Letztlich sind Klein(st)parteien der manifeste Ausdruck höherer Individualisierung von politischen Präferenzen, die den (größeren) Parteien deutlich zu schaffen macht (Mair und van Biezen 2001, S. 14).

Bei Untersuchungen der radikalen Rechten konnte zudem festgestellt werden, dass die Teilnahme in einer Partei eher nicht von charismatischen Führungspersönlichkeiten abhängt (Whiteley et al. 2019, S. 10), was tendenziell dafür spricht, dass vor allem die Ideologie der entscheidende Faktor für diese Mitglieder ist. Dass die Ideologie in diesem Rahmen eine gewichtige Rolle in Bezug auf die Aktivität einnimmt, konnte ebenso gezeigt werden, wenngleich mit abnehmendem Effekt ab einer gewissen Stufe der Radikalität (Whiteley et al. 2019, S. 7). Grundsätzlich ist für die anderen kleinen Parteien Ähnliches zu erwarten. Im Falle der ÖDP konnte dieser Effekt bei den Aktiven allerdings nicht nachgewiesen werden (Biehl und Kranenpohl 2011). Insofern kommt es hier durchaus auch auf die jeweilige Partei an.

Da sowohl die Entwicklung neuer politischer Ideen als auch das Bewahren politischer Traditionen auf die Ideologie der Partei abzielen und diese allgemein einen wichtigen Faktor für den Parteibeitritt darstellen, sollen diese Funktionen zur zweiten Hypothese zusammengeführt werden.

H2 Mitglieder von Klein(st)parteien sind ihren politischen Idealen stärker verpflichtet als Mitglieder von etablierten Parteien.

Klein(st)parteien und ihre Mitglieder sehen sich zudem als Kontrolleure der Mächtigen. Ihr Handlungsspielraum in dieser Funktion ist zwar begrenzt, je- 
doch durchaus vorhanden. Aufgrund der relativ großen Gewaltenverschränkung in Deutschland sind die Kontrollmechanismen vornehmlich durch die föderale Struktur und das Verfassungsgericht gegeben und weniger beim Zusammenspiel von Legislative und Exekutive auf Parlamentsebene. Auch die Medien erfüllen Kontrollfunktionen, sind jedoch teilweise durch informelle und formelle Beziehungen ebenso abhängig von Parteien. Klein(st)parteien dienen insofern als Kontrolleure der Mächtigen, als sie beispielsweise bei weitreichenden Fehlern der etablierten Parteien die unzufriedenen Wähler kanalisieren können (van den Boom 1999, S. 272-274).

In diesen Klein(st)parteien findet letztlich relativ normale Parteiarbeit statt. Das heißt, dass zum einen Resolutionen zu aktuellen Fragen der Zeit beschlossen werden und zum anderen der Anspruch besteht, auf die Politik Einfluss zu nehmen. Klein(st)parteien sind dementsprechend als „Parteien im Wartestand“ anzusehen. Sie stehen in Konkurrenz zu denjenigen Parteien, die derzeit Verantwortung tragen, wodurch sie den Handlungsrahmen der politischen Entscheidungsträger einschränken (van den Boom 1999, S. 272-274). Diese Parteien stehen daher im ,Schatten der Macht" und warten darauf, aus diesem herauszutreten, ausgelöst beispielsweise durch Fehltritte der großen Parteien.

Da die Parteien jedoch nicht in Machtpositionen sind, scheint es bei ihren Mitgliedern ein äußerst ausgeprägtes Pflichtgefühl zu geben, sich trotzdem in einer Partei zu engagieren. Schließlich ist die unmittelbare Wirksamkeit doch relativ gering. Sich dennoch in einer solch kleinen Partei zu engagieren, dürfte dementsprechend aus einem Pflichtgefühl dem System gegenüber gespeist sein. Ein ähnliches Bild ergab sich bereits bei der Untersuchung von Multi-Speed-Membership-Parteien ${ }^{8}$ (Gomez et al. 2019). Hier ist ebenfalls fraglich, ob mit der Aufnahme einer formalen Mitgliedschaft eine erhöhte Wirksamkeit einhergeht. Dennoch zeigte sich, dass der Motivationsfaktor, einen Beitrag zum Funktionieren der Demokratie zu leisten, für den letztlichen Beitritt eine gewichtige Rolle einnimmt. Daraus ergibt sich die dritte Hypothese.

H3 Mitglieder von Klein(st)parteien stellen ihre Bürgerpflicht und ihren Beitrag zum Funktionieren der Demokratie stärker in den Vordergrund.

Klein(st)parteien dienen auch als Orte der „Vereinsmeierei“ (van den Boom 1999). Die Mitglieder dieser Parteien haben ein ausgeprägtes Bedürfnis nach Gemeinschaft und Führung, welches die großen Parteien nicht befriedigen können. Dies wird auch als ein Grund angeführt, warum diese Klein(st)parteien in der Regel ausgeprägte regionale Schwerpunkte aufweisen (für beispielsweise die ÖDP siehe Kranenpohl 2008, S. 49). Die Parteimitgliedschaft wird dementsprechend als eine weitere Form des Vereinslebens angesehen. Anstelle des Engagements beispielsweise in einem Taubenzüchterverein tritt mit der Parteimitgliedschaft eine etwas anspruchsvollere Tätigkeit. Abhängig von der Größe und dem Organisationsgrad sind einige Mitglieder auch durch die Möglichkeit motiviert, sich als bezahlter Mitarbeiter für die Partei zu engagieren. Fällt die Bezahlung aus, stehen ausgesprochener

\footnotetext{
8 Multi-Speed-Membership-Parteien zeichnen sich durch eine geringere Formalisierung der ,Mitgliedschaft“ aus. So ist es neben der normalen Mitgliedschaft unter anderem möglich, sich als „Unterstützer“ eintragen zu lassen. Siehe dazu beispielsweise Hooghe und Kölln (2020).
} 
Dank, das Vertrauen, das einem entgegengebracht wird und auch die Achtung, die man durch das Engagement erfährt, im Vordergrund. Zudem ist es in solchen Parteien deutlich einfacher, einen Posten zu besetzen, als es bei größeren Parteien der Fall ist (van den Boom 1999). Eine derartige Ämtermotivation findet sich häufiger bei kleinen Parteien (Müller und Traub 2004). Obwohl es nur Ämter bei kleinen Parteien sind, sind auch diese Posten mit gewissem Ansehen und Prestige, zumindest unter den anderen Mitgliedern der eigenen Partei, verbunden. Teilweise haben die aktiven Mitglieder auch nichts anderes als die Arbeit in ihrer Klein(st)partei (van den Boom 1999, S. 274-279). Doch nicht nur der Anreiz, selbst ein Amt bekleiden zu können, ist von Bedeutung. Schon die Möglichkeit, Einfluss auf die Wahl des politischen Führungspersonals und die Ausrichtung der Partei zu nehmen, ist nicht zu unterschätzen (Achury et al. 2020, S. 58). Hierbei sind die Einflussmöglichkeiten bei einer Klein(st)partei natürlich deutlich höher als bei einer großen Partei.

Bei den größeren Parteien hat sich diesbezüglich gezeigt, dass die Mitglieder ihren Einfluss in der eigenen Partei doch als relativ gering empfinden (Vetter 2004). Dieses Einflussgefühl dürfte bei den Klein(st)parteien deutlich stärker ausgeprägt sein, da die tatsächlichen Einflussmöglichkeiten des Einzelnen bei weniger Parteimitgliedern höher sind. Schon bei den kleinen unter den im Parlament vertretenen Parteien, wie den Grünen, ließ sich der Effekt des erhöhten Einflussgefühls in der eigenen Partei feststellen (Vetter 2004). Das bedeutet, dass die Parteimitgliedschaft hier mehr Befriedigung verschaffen kann, weil das „Sich-einbringen“ auch eher in Resultaten münden kann (Vetter 2004). Im Einklang damit ist die Größe der Partei auch ein ausschlaggebender Faktor dafür, wie aktiv die Mitglieder letztlich sind (Weldon 2006). Darüber hinaus wurde bereits bei Untersuchungen zur Aktivität in einer radikal rechten Partei festgestellt, dass die soziale Vernetzung eine wichtige Quelle der Motivation darstellt (Whiteley et al. 2019). Ein ähnliches Bild zeigte sich ebenfalls bei der ÖDP in Deutschland (Biehl und Kranenpohl 2011). Es ist jedoch zu erwarten, dass nicht nur die Aktivität, sondern bereits der Beitritt von diesem Motivationsfaktor abhängt. Grundsätzlich zeigt sich zudem im Hinblick auf die Multi-Speed-Membership-Parteien, dass die selektiven, prozessbezogenen Anreize eine wichtige Quelle für den Beitritt, anstelle des Sympathisantenstatus, darstellen (Gomez et al. 2019).

Inaktive Mitglieder gibt es natürlich ebenso bei den Klein(st)parteien. Hier gestaltet sich die Frage nach der Motivation etwas schwieriger. Es wird angeführt, dass diese aus Gründen der Beteiligung am kollektiven Gut der Interessenvertretung Mitglied sind und bleiben. Hierdurch wird das Gefühl evoziert, man „,mache ja etwas“ (van den Boom 1999, S. 274-279).

Daraus lässt sich schließen, dass die Parteiarbeit selbst schon Befriedigung für die Mitglieder beschafft, sei dies in Form von Ämtern und dem damit verbundenen Ansehen oder allgemein durch das ,,in der Partei sein“. Aus diesen Funktionen ergibt sich die vierte Hypothese.

H4 Mitgliedern von Klein(st)parteien verschafft die Mitgliedschaft mehr Befriedigung (sie erwarten Ämter; die Mitgliedschaft an sich verschafft mehr Befriedigung).

Zusammenfassend erwarten wir also bei Klein(st)parteien stärkere Ausprägungen der ideologischen, der altruistischen, der selektiven, ergebnis- und prozessbezogenen 
und der expressiven Anreize. Die anderen klassischen Anreize, die dem GeneralIncentives-Modell entstammen, erwarten wir auf einem vergleichbaren Level wie bei den größeren Parteien und spielen deshalb in der folgenden Analyse nur eine marginale Rolle.

\section{Methode und Daten}

Eine Gegenüberstellung des parteipolitischen Engagements von Mitgliedern der Klein(st)parteien und Mitgliedern der etablierten Parteien lässt sich nicht auf Grundlage von Bevölkerungsbefragungen realisieren. Da in Deutschland lediglich um die 2\% der Wahlberechtigten Parteimitglieder sind (Niedermayer 2020; Daten von 2009), enthalten derartige Umfragen, zumindest bei ausbleibender disproportionaler Schichtung, nur vereinzelt Mitglieder von kleinen Parteien. In Kombination mit der Notwendigkeit für eine detaillierte Erfassung der Einstellungen der Befragten zur Mitarbeit in der eigenen Partei resultiert daraus für die vorliegenden Analysen der Rückgriff auf eine dezidierte Parteimitgliederbefragung. Es handelt sich dabei um die Deutsche Parteimitgliederstudie 2009 (Spier et al. 2011). Während die im Rahmen dieses Projekts durchgeführte postalische Befragung aufgrund ihrer Beschränkung auf Mitglieder parlamentarisch vertretener Parteien für unseren Untersuchungsgegenstand ungeeignet ist, verfügt die Telefonbefragung als die zweite Komponente dieser Studie über die notwendigen Eigenschaften.

Im Rahmen der telefonischen Befragung wurden von Infratest dimap zwischen dem 14.04. und dem 30.05.2009 Interviews mit 800 Parteimitgliedern und 827 Personen, die noch nie Mitglied einer Partei waren, durchgeführt. Die Grundgesamtheit bildeten deutsche Staatsbürger, die mindestens 18 Jahre alt sind, in einem privaten Haushalt leben und je nach Stichprobe aktuelles Parteimitglied oder parteipolitisch unerfahren sind. Die Ziehung dieser Zufallsstichproben erfolgte auf Grundlage des TNS Infratest Telefon-Master-Samples (ITMS). ${ }^{9}$ Um die hohe Anzahl der Parteimitglieder sicherzustellen, mussten zunächst Screening-Interviews erfolgen. Erst in einem zweiten Schritt wurden die Wiederbefragungswilligen umfassend interviewt. Die so getätigte Erhebung gibt ,Aufschluss über die sozialstrukturelle, psychografische und aktivitätsbezogene Zusammensetzung der Mitgliedschaft der deutschen Parteien sowie über die Motive des Parteibeitritts, der innerparteilichen Aktivität und des Parteiaustritts“ (Rohrbach 2013, S. 145). Dabei variierte das Fragenprogramm zwischen den Parteimitgliedern und den parteipolitisch Unerfahrenen lediglich dann, wenn es inhaltlich geboten war. Dies betrifft naturgemäß vor allem Fragen zur Partizipation in politischen Parteien. Daraus folgt für die eigenen Analysen die

\footnotetext{
9 Das ITMS ist so angelegt worden, dass es die Ziehung verzerrungsfreier Stichproben ohne Klumpeneffekte ermöglicht. Die Verzerrungsfreiheit wird u. a. dadurch gewährleistet, dass zur Vermeidung des ,Notat-Home-Bias“ nicht erreichte Haushalte bis zu 12-mal in großer zeitlicher Variation angerufen werden. Nach Ziehung der Stichprobe von Telefonhaushalten auf Grundlage des ITMS wurde die innerhalb eines Haushalts zu befragende Person per Schwedenschlüssel ermittelt.
} 
Erläuterung etwaiger Unterschiede bei dem gewählten Fragelaut sowie teilweise der Verzicht auf Vergleiche der Parteimitglieder mit der übrigen Bevölkerung. ${ }^{10}$

Im Fortlauf der Untersuchung untergliedern wir die aktuellen Mitglieder nach Zugehörigkeit zu Klein(st)parteien und etablierten Parteien. Wie sich die Befragten auf die einzelnen Parteien verteilen, ist Tab. 9 im Online-Anhang zu entnehmen. Insgesamt sind 31 Mitglieder von Klein(st)parteien in den Daten enthalten. Sie verteilen sich ausgewogen auf 16 verschiedene Parteien diverser Parteienfamilien. Insofern ist der programmatischen Bandbreite von Klein(st)parteien in der Stichprobe Rechnung getragen. Dennoch ist bei der geringen Fallzahl keine perfekte Repräsentation aller Strömungen der Klein(st)parteien möglich. Dies ist für die Analysen weitgehend unproblematisch, da sich unsere Hypothesen im Kern aus dem Definitionskriterium einer Klein(st)partei - der beschränkten parlamentarischen Repräsentanz herleiten. Lediglich bei einzelnen Variablen, wie dem Abstand zur politischen Mitte oder der Bereitschaft zu verschiedenen Formen politischer Partizipation, erscheint es plausibel, dass die spezifische Zusammensetzung der Klein(st)parteimitglieder nach verschiedenen Parteifamilien bedeutsam sein könnte. Da alle Hypothesen anhand mehrerer, verschieden gelagerter, Variablen überprüft werden, ist hierdurch allerdings keine unserer Kernaussagen berührt.

Wir haben in unseren Analysen Mitglieder der Freien Wähler beziehungsweise unabhängiger Wählergemeinschaften nicht berücksichtigt. Ursächlich für den Ausschluss der elf betroffenen Personen ist, dass die Bundesvereinigung der Freien Wähler erst im Befragungsjahr als Partei gegründet wurde. ${ }^{11}$ Es lässt sich argumentieren, dass diese Befragten sich offenbar trotzdem (bereits) als Parteimitglieder verstanden. Schließlich bejahten sie beim Screening-Interview die Frage nach einer Parteimitgliedschaft und waren in der Folge auch dazu bereit, sich zu dieser (vermeintlichen) Parteimitgliedschaft im Detail befragen zu lassen. Aufgrund dieses Sachverhalts haben wir unsere Analysen zusätzlich unter Berücksichtigung der betroffenen Befragten in der Gruppe der Klein(st)parteimitglieder durchgeführt. Dabei erwiesen sich die im Folgenden berichteten Ergebnisse als robust. Es sind nur selten nennenswerte Veränderungen der Mittelwerte aufgetreten, wobei keine unse-

\footnotetext{
10 Wenn wir im Weiteren auf die „Noch-Nie-Parteimitglieder“ als Vergleichsgruppe zu den Mitgliedern von Klein(st)parteien und etablierten Parteien rekurrieren, verwenden wir der Einfachheit halber den Begriff der Bevölkerung. Dies enthält die Unschärfe, dass aktuelle Parteimitglieder zur Bevölkerung gehören, hier aber bewusst nicht enthalten sind. Genauso wenig sind die, gemäß Screening-Interviews der verwendeten Telefonbefragung, gut $5 \%$ der Bevölkerung aufgenommen, die ehemalige Parteimitglieder sind. Da man zumindest bei den ehemaligen Parteimitgliedern auch für eine Berücksichtigung in den Analysen plädieren kann, haben wir alternativ auf Daten zu 800 ehemaligen Mitgliedern zurückgegriffen, die eine dritte Stichprobe im Rahmen der Telefonbefragung der Parteimitgliederstudie bilden. Aufgrund ihres geringen Bevölkerungsanteils hat die Inklusion dieser Personengruppe nur einen geringen Einfluss auf unsere Befunde. Die einzigen systematischen Unterschiede relevanter Größe zeigen sich bei den selektiven Anreizen (besonders bei der Übernahme von Parteiämtern, dem Knüpfen beruflicher Kontakte und der Teilnahme an geselligen Veranstaltungen). Ehemalige Mitglieder scheinen bezüglich dieser persönlichen Vorteile oftmals desillusioniert zu sein. In manchen Fällen könnte dies den Parteiaustritt forciert haben.

11 Dass die Freien Wähler durchaus auch schon vor 2009 als Grenzfall wahrgenommen wurden, zeigt sich beispielsweise in folgender Argumentation: ,Indem [die Freien Wähler] als Organisationen auf Dauer angelegt sind und sich um politische Mandate bei Wahlen bewerben, erfüllen sie den Status einer Partei, auch wenn das Bundesparteiengesetz und einige Landeswahlgesetze die Teilnahme an Bundestags- beziehungsweise Landtagswahlen dafür zur Voraussetzung machen“ (Wehling 2007, S. 288).
} 
rer Einschätzungen zur Validität der Hypothesen von der Inklusion der Befragten abhängt.

Für die Untersuchung der einzelnen Hypothesen greifen wir schließlich auf unterschiedliche Indikatoren zurück, die zur Hypothese passend erscheinen. Wir stellen also aufgrund des spärlichen Wissensstandes zur Mitgliedschaft von Klein(st)parteien möglichst umfassende Informationen bereit. Auch soll die Berücksichtigung vieler Messgrößen der geringen Fallzahl bei Klein(st)parteimitgliedern entgegenwirken. Etwaige, durch einige wenige Befragte bedingte, Ausreißer bei einzelnen Items haben hierdurch keine verzerrte Beurteilung von Hypothesen zur Folge.

Die Überprüfung unserer Hypothesen geschieht auf Grundlage von Mittelwertvergleichen. Zu diesem Zweck ist der Gebrauch zweiseitiger t-Tests üblich. Wir haben allerdings festgestellt, dass in unseren Daten mit der Normalverteilung eine der Anwendungsvoraussetzungen nicht immer erfüllt ist. Zwar sind t-Tests gegen Verletzungen der zugrundeliegenden Annahmen robust. Dies gilt allerdings nicht bei so kleinen Fallzahlen wie sie in dieser Studie bei den Klein(st)parteimitgliedern vorliegen. Dementsprechend greifen wir stattdessen auf Mann-Whitney-U-Tests zurück, die keine Normalverteilung voraussetzen. Ein weiteres Problem bei Verwendung von t-Tests hätte darin bestanden, dass ein Teil unserer Variablen maximal als quasimetrisch begriffen werden kann. Dies betrifft im Besonderen die für Hypothese 1 maßgeblichen Messungen politischer Unterstützung, die lediglich fünf Merkmalsausprägungen aufweisen. Da der Mann-Whitney-U-Test speziell für ordinalskalierte Variablen entwickelt wurde, ist diese Konstellation nun unproblematisch. ${ }^{12}$

\section{Analyse}

Ganz im Einklang mit Hypothese 1, fungieren Klein(st)parteien gemäß unseren Analysen tatsächlich als ein Sammelbecken der Unzufriedenen. Die Mittelwertvergleiche in Tab. 4 zeigen schließlich, dass die Mitglieder von Klein(st)parteien deutlich unzufriedener als die Mitglieder der großen Parteien sind. Dies trifft sowohl auf die Zufriedenheit mit der Demokratie und der Regierung als auch auf die Zufriedenheit mit den Parteien zu, was auch bereits für Mitglieder von Randparteien herausgearbeitet wurde (Höhne 2018). Die Mitglieder der Klein(st)parteien sind ferner deutlich unzufriedener mit dem System als die Nichtparteimitglieder. Hieraus lässt sich schließen, dass sowohl eine hohe als auch eine besonders geringe Zufriedenheit mit dem System einen starken Ausschlag dafür geben, in einer Partei Mitglied zu werden. Der erstgenannte Fall begünstigt den Eintritt in eine große Partei, im zweiten Fall fällt die Wahl eher auf eine Klein(st)partei.

Unzufriedenheit kann u. a. durch die Wahrnehmung einer geringen Beeinflussbarkeit des politischen Systems bedingt sein. Insofern überrascht es nicht, dass auch bei der Wahrnehmung der Responsivität des Systems, der sogenannten ,external

12 Zudem haben wir bei allen die Klein(st)parteimitglieder einschließenden Vergleichen per Levene-Test auf Varianzhomogenität geprüft. Bei Vorliegen von Heteroskedastizität weisen wir zusätzlich zum MannWhitney-U-Test t-Tests mit nach der Satterthwaite-Methode ermittelten Freiheitsgraden aus. 
Tab. 4 Indikatoren politischer Unterstützung bei Nichtparteimitgliedern, Mitgliedern von Klein(st)parteien und Mitgliedern von etablierten Parteien

\begin{tabular}{|c|c|c|c|c|c|c|c|c|c|}
\hline \multirow{3}{*}{$\begin{array}{l}\text { Variablen (Range) } \\
\text { Zufriedenheit mit der } \\
\text { Demokratie (1-5) }\end{array}$} & \multicolumn{3}{|c|}{ Mittelwerte } & \multicolumn{6}{|c|}{ Mittelwertdifferenzen } \\
\hline & \multirow{2}{*}{$\frac{\mathrm{GP}}{3,0}$} & \multirow{2}{*}{$\frac{\mathrm{KP}}{2,2}$} & \multirow{2}{*}{$\frac{\mathrm{Bev}}{2,8}$} & \multicolumn{2}{|c|}{ GP-KP } & \multicolumn{2}{|c|}{ GP-Bev } & \multicolumn{2}{|c|}{$\mathrm{KP}-\mathrm{Bev}$} \\
\hline & & & & 0,8 & $* * * 1$ & 0,2 & $* * *$ & $-0,6$ & $* * *$ \\
\hline $\begin{array}{l}\text { Zufriedenheit mit den } \\
\text { Parteien (1-5) }\end{array}$ & 2,5 & 2,0 & 2,3 & 0,5 & $* * *$ & 0,2 & $* * *$ & $-0,3$ & $*$ \\
\hline $\begin{array}{l}\text { Zufriedenheit mit der } \\
\text { Regierung (1-5) }\end{array}$ & 2,6 & 1,9 & 2,5 & 0,7 & $* * *$ & 0,1 & $* * *$ & $-0,6$ & $* * *$ \\
\hline $\begin{array}{l}\text { Internal political } \\
\text { efficacy }(1-5)\end{array}$ & 4,0 & 4,0 & 3,2 & 0,1 & 1 & 0,8 & $* * *$ & 0,7 & $* * * 1$ \\
\hline $\begin{array}{l}\text { External political } \\
\text { efficacy }(1-5)\end{array}$ & 3,0 & 2,2 & 2,5 & 0,8 & $* * *$ & 0,4 & $* * *$ & $-0,4$ & $* *$ \\
\hline $\begin{array}{l}\text { Institutionenvertrauen } \\
(1-5)\end{array}$ & 2,9 & 2,4 & 2,7 & 0,5 & $* * * 1$ & 0,1 & $* * *$ & $-0,3$ & $* * *$ \\
\hline
\end{tabular}

GP Mitglieder etablierter Parteien: $n=733-747, K P$ Mitglieder Klein(st)parteien: $n=29-31$, Bev Bevölkerung: $n=817-827$

${ }^{+} p<0,10, * p<0,05, * * p<0,01, * * * p<0,001$

Die Einschätzungen zur Verschiedenheit der Mittelwerte basieren auf dem Mann-Whitney-U-Test. Bei Angabe von ," " verwendeten wir zusätzlich einen zweiseitigen t-Test mit Freiheitsgraden nach der Satterthwaite-Methode, da gemäß dem Levene-Test in den betreffenden Fällen Heteroskedastizität besteht. Für die in dieser Tabelle so gekennzeichneten Fälle liegen gemäß beiden Tests jeweils dieselben Signifikanzniveaus vor

efficacy“, deutliche Unterschiede zwischen Mitgliedern der großen Parteien und der Klein(st)parteien bestehen, was auch bereits die Ergebnisse zur ÖDP angedeutet haben (Biehl und Kranenpohl 2011). Es zeigt sich, dass Klein(st)parteimitglieder deutlich kritischere Ansichten vertreten als dies bei Parteimitgliedern der großen Parteien, aber auch der allgemeinen Bevölkerung der Fall ist. Klein(st)parteimitglieder wollen dementsprechend nicht, dass die Politik allein den Interessenvertretern überlassen wird und sie sind mit diesen auch nicht sonderlich zufrieden. Des Weiteren sehen sie direktdemokratische Elemente als wichtiger an und wünschen sich diese im Vergleich zu Mitgliedern großer Parteien und zur allgemeinen Bevölkerung deutlich mehr. Das gleiche Bild zeichnet sich beim Institutionenvertrauen ab, welches bei Mitgliedern von Klein(st)parteien merklich geringer ausgeprägt ist als bei Mitgliedern etablierter Parteien und der allgemeinen Bevölkerung. Dies unterfüttert noch einmal die Annahme, dass es zwei Schwellpunkte gibt, die zu einer Parteimitgliedschaft führen. Zum einen die generelle Zufriedenheit mit der Responsivität (die zur Mitgliedschaft in einer großen Partei beiträgt) und zum anderen die starke Unzufriedenheit mit dieser (welche zur Mitgliedschaft in einer Klein(st)partei beiträgt). Beide Punkte sprechen eher für eine Mitgliedschaft, wohingegen eine relative Indifferenz schließlich von einer Mitgliedschaft abhält.

Was die ,internal efficacy“, also die Einschätzung des eigenen politischen Vermögens, angeht, gibt es kaum Unterschiede zu den großen Parteien. Klein(st)parteimitglieder trauen sich genauso wie die Mitglieder der großen Parteien zu, eine aktive Rolle in der politischen Arbeit einzunehmen und sind ebenso der Ansicht, dass die Politik nicht zu kompliziert und unverständlich ist. Lediglich was die Einschätzung 
Tab. 5 Relevanz politischer Ideale für Nichtparteimitglieder, Mitglieder von Klein(st)parteien und Mitglieder von etablierten Parteien

\begin{tabular}{|c|c|c|c|c|c|c|c|c|c|}
\hline \multirow{3}{*}{$\begin{array}{l}\text { Variablen (Range) } \\
\text { Bereitschaft zum Kampf } \\
\text { für parteiinternen Kurs- } \\
\text { wechsel (1-5) }\end{array}$} & \multicolumn{3}{|c|}{ Mittelwerte } & \multicolumn{6}{|c|}{ Mittelwertdifferenzen } \\
\hline & \multirow{2}{*}{$\frac{\text { GP }}{4,2}$} & \multirow{2}{*}{$\frac{\mathrm{KP}}{3,5}$} & \multirow{2}{*}{$\frac{\operatorname{Bev}}{-}$} & \multicolumn{2}{|c|}{$\mathrm{GP}-\mathrm{KP}$} & \multicolumn{2}{|c|}{$\mathrm{GP}-\mathrm{Bev}$} & \multicolumn{2}{|c|}{$\mathrm{KP}-\mathrm{Bev}$} \\
\hline & & & & 0,7 & $* * 1$ & - & - & - & - \\
\hline $\begin{array}{l}\text { Abstand zum Mittel- } \\
\text { punkt der Links-Rechts- } \\
\text { Skala (0-10) }\end{array}$ & 1,8 & 2,7 & 1,4 & $-0,9$ & $* 1$ & 0,4 & $* * *$ & 1,3 & $* * * 1$ \\
\hline \multicolumn{10}{|c|}{ Ideologische Anreize (0-10) } \\
\hline $\begin{array}{l}\text { Einstehen für politische } \\
\text { Überzeugung }\end{array}$ & 8,0 & 8,5 & 7,1 & $-0,6$ & $*$ & 0,8 & $* * *$ & 1,4 & $* * *$ \\
\hline $\begin{array}{l}\text { Stärkung politischer } \\
\text { Ideale }\end{array}$ & 6,1 & 7,0 & 5,4 & $-0,9$ & + & 0,7 & $* * *$ & 1,6 & $* * *$ \\
\hline \multicolumn{10}{|c|}{ Kollektive politische Anreize (0-10) } \\
\hline $\begin{array}{l}\text { Durchsetzung politi- } \\
\text { scher Ziele }\end{array}$ & 5,9 & 6,6 & 5,6 & $-0,7$ & & 0,3 & $*$ & 1,0 & $* 1$ \\
\hline
\end{tabular}

GP Mitglieder etablierter Parteien: $n=736-746, K P$ Mitglieder Klein(st)parteien: $n=30-31$, Bev Bevölkerung: $n=789-800$

${ }^{+} p<0,10, * p<0,05, * * p<0,01, * * * p<0,001$

Die Einschätzungen zur Verschiedenheit der Mittelwerte basieren auf dem Mann-Whitney-U-Test. Bei Angabe von „, “ verwenden wir zusätzlich einen zweiseitigen t-Test mit Freiheitsgraden nach der Satterthwaite-Methode, da gemäß dem Levene-Test in den betreffenden Fällen Heteroskedastizität besteht. Dabei ergibt sich gegenüber den in der Tabelle dokumentierten Befunden des Mann-Whitney-U-Tests ein Unterschied: Für die erhöhte Bereitschaft von Mitgliedern großer Parteien zum Kampf für einen parteiinternen Kurswechsel wird bei Nutzung des t-Tests das höchste ausgewiesene Signifikanzniveau erreicht

von wichtigen politischen Fragen angeht, schätzen sich Klein(st)parteimitglieder leicht schlechter ein, als es die Mitglieder großer Parteien tun. Demnach trauen sich Klein(st)parteimitglieder also grundsätzlich zu, politische Fragen zu durchschauen und auch am politischen Prozess teilzunehmen, ganz im Gegensatz zu der allgemeinen Bevölkerung. Dies stimmt auch mit den meisten Untersuchungen zu Parteimitgliedern überein, die herausstellten, dass eben dies ein bedeutender Faktor ist, der eine Parteimitgliedschaft begünstigt (Weber 2020; Hoffmann und Springer 2019). Hierbei ist es dementsprechend unerheblich, ob das letztlich in einer Mitgliedschaft bei den großen Parteien oder bei den Klein(st)parteien mündet.

Hypothese 1 kann somit bestätigt werden. Mitglieder von Klein(st)parteien sind deutlich unzufriedener mit dem System als Mitglieder von großen Parteien. Ebenso sind sie unzufriedener als die allgemeine Bevölkerung. Dies liegt unter anderem auch daran, dass diese Mitglieder wenig Vertrauen in die Institutionen haben und auch die Beeinflussbarkeit und Responsivität des Systems deutlich negativer bewerten.

Tendenziell deuten unsere Daten auch auf die Validität von Hypothese 2 hin (siehe Tab. 5), weisen doch die Klein(st)parteimitglieder durchgehend höhere Werte auf, was die ideologischen und kollektiven, politischen Anreize betrifft. Sie haben also stärker als Mitglieder der großen Parteien die Erfahrung gemacht, durch ihre Parteiarbeit für die eigenen politischen Einstellungen einzustehen, bestimmte politische Ideale zu stärken und damit auch einen Beitrag zur Durchsetzung politischer Ziele zu leisten. Die vorhandenen Differenzen sind vor dem Hintergrund bemerkenswert, 
dass bereits Anhänger der größeren Parteien diese möglichen Folgen der Parteimitgliedschaft für deutlich realistischer halten als die übrige Bevölkerung. Bei den ideologischen Anreizen handelt es sich schließlich sogar um die durch Parteimitglieder am zweithöchsten bewertete Dimension des GIM, was im Einklang mit bisherigen Ergebnissen zu Parteimitgliedern steht (Hoffmann und Springer 2019; Weber 2020). In das Bild einer stärkeren ideologischen Ausrichtung passt ferner, dass die Kleins(st)parteimitglieder politisch extremer als die beiden Vergleichsgruppen eingestellt sind. Hier ist allerdings erneut Vorsicht bei der Interpretation anzumahnen, da gerade bezüglich der ideologischen Positionierung zu befürchten ist, dass die spezifische Zusammensetzung der Klein(st)parteimitglieder hinsichtlich verschiedener Parteifamilien unsere Ergebnisse geprägt haben kann (siehe auch Kap. 3). Es ist also unklar, ob diese Differenz bei einer anderen Komposition erhalten bliebe.

Scheinbar im Widerspruch zu unserer Hypothese einer erhöhten Ideologiegetriebenheit steht dagegen der deutlich größere Widerwillen bei Klein(st)parteimitgliedern, für einen Kurswechsel innerhalb der eigenen Partei zu kämpfen. Eine mögliche Erklärung besteht darin, dass ein solcher Konflikt in einer Klein(st)partei nicht notwendig ist, da sich hier im Allgemeinen vermutlich homogenere Gruppen zusammenfinden als dies in parlamentarisch vertretenen Parteien der Fall ist. Mit van den Boom (1999) lässt sich ferner argumentieren, dass Mitglieder in Klein(st)parteien den parteiinternen Positionskämpfen bewusst ausweichen wollen.

Insgesamt zeigt sich, dass Ideologie eine große Rolle spielt, wenn es darum geht, Parteimitglied zu werden. Dies gilt für die Klein(st)parteimitglieder erwartungsgemäß noch stärker als für die Mitglieder großer Parteien.

Aus der rational-choice-basierten Forschung zu Wahlbeteiligung ist das Wahlparadoxon bekannt (Ferejohn und Fiorina 1974). Die scheinbare Unvernunft, Aufwand für die Wahlteilnahme zu betreiben, obgleich die eigene Stimme nur im sehr unwahrscheinlichen Fall wahlentscheidend sein wird, löst sich bei Akzeptanz eines weiten Rationalitätsbegriffs dadurch auf, dass Menschen ihre Stimmabgabe als Beitrag zum Funktionieren der Demokratie betrachten. Auch die individuelle Parteimitgliedschaft hat in der Mehrheit der Fälle keine Auswirkungen auf die personelle und inhaltliche Ausrichtung einer Partei. Entsprechend wurde auch in der ursprünglichen Konzeption des General-Incentives-Modells (GIM) bereits die Überlegung berücksichtigt, dass Bürger sich einer Partei anschließen, weil sie eine solche Mitgliedschaft als Bürgerpflicht betrachten. Die Items zur Operationalisierung dieser sogenannten altruistischen Anreize erfahren hinter den ideologischen Anreizen die höchste Zustimmung unter den Parteimitgliedern. Es ist naheliegend, dass hier nennenswerte Differenzen zu der übrigen Bevölkerung bestehen, entspräche eine besonders positive Bewertung der betreffenden Items durch Nichtmitglieder doch der Selbstbezichtigung der Nichterfüllung einer Bürgerpflicht. Von unseren Erwartungen weicht dagegen ab, dass Mitglieder etablierter Parteien altruistischen Anreizen eine ebenso hohe Wertschätzung entgegenbringen wie Klein(st)parteimitglieder (Tab. 6). Ursächlich für die ausbleibende Differenz könnte die soeben skizzierte Konstellation sein, dass ungeachtet der Parteigröße die Mehrheit der Mitglieder keinen Einfluss auf Politikgestaltung ausübt.

Erweitert man die Perspektive auf die wahrgenommene Bedeutsamkeit von politischer Teilhabe insgesamt, zeigt sich auch hier, dass Parteimitglieder politischer Parti- 
Tab. 6 Einstellungen zu politischer Partizipation und altruistische Anreize bei Nichtparteimitgliedern, Mitgliedern von Klein(st)parteien und Mitgliedern von etablierten Parteien

\begin{tabular}{|c|c|c|c|c|c|c|c|c|c|}
\hline \multirow{3}{*}{$\begin{array}{l}\text { Variablen (Range) } \\
\text { Anzahl Formen poli- } \\
\text { tischen Protests, die } \\
\text { Befragter erwägt }(0-12)\end{array}$} & \multicolumn{3}{|c|}{ Mittelwerte } & \multicolumn{6}{|c|}{ Mittelwertdifferenzen } \\
\hline & \multirow{2}{*}{$\frac{\mathrm{GP}}{8,0}$} & \multirow{2}{*}{$\frac{\mathrm{KP}}{8,6}$} & \multirow{2}{*}{$\frac{\mathrm{Bev}}{7,1}$} & \multicolumn{2}{|c|}{$\mathrm{GP}-\mathrm{KP}$} & \multicolumn{2}{|c|}{ GP - Bev } & \multicolumn{2}{|c|}{$\mathrm{KP}-\mathrm{Bev}$} \\
\hline & & & & $-0,6$ & $*$ & 0,9 & $* * *$ & 1,5 & $* * *$ \\
\hline $\begin{array}{l}\text { Gesell. Relevanz po- } \\
\text { litischer Partizipation } \\
(1-10)\end{array}$ & 7,6 & 7,6 & 6,5 & 0,0 & & 1,1 & $* * *$ & 1,1 & $* *$ \\
\hline \multicolumn{10}{|c|}{ Altruistische Anreize (0-10) } \\
\hline $\begin{array}{l}\text { Beitrag zum Funktionie- } \\
\text { ren der Demokratie }\end{array}$ & 6,5 & 6,5 & 5,6 & 0,0 & & 0,9 & $* * *$ & 0,9 & + \\
\hline $\begin{array}{l}\text { Erfüllung einer Bürger- } \\
\text { pflicht }\end{array}$ & 6,8 & 7,2 & 5,4 & $-0,5$ & & 1,3 & $* * *$ & 1,8 & $* * *$ \\
\hline
\end{tabular}

GP Mitglieder etablierter Parteien: $n=743-747, K P$ Mitglieder Klein(st)parteien: $n=30-31$, Bev Bevölkerung: $n=793-827$

${ }^{+} p<0,10, * p<0,05, * * p<0,01, * * * p<0,001$

Die Einschätzungen zur Verschiedenheit der Mittelwerte basieren auf dem Mann-Whitney-U-Test

zipation einen hohen gesellschaftlichen Nutzen zuweisen, der die Beurteilung durch die Bevölkerung deutlich übersteigt. Erneut sind dabei keine Unterschiede zwischen Klein(st)parteimitgliedern und Mitgliedern etablierter Parteien beobachtbar. Erst bei Betrachtung der Bereitschaft zur Teilnahme an anderen Partizipationsformen zeigt sich, dass Klein(st)parteimitglieder in ihrem Beteiligungsrepertoire breiter als Mitglieder etablierter Parteien und vor allem breiter als die Bevölkerung aufgestellt sind. ${ }^{13}$ Insofern bestätigt sich, dass die Wahrscheinlichkeit, an Protestaktionen teilzunehmen, bei Parteimitgliedern insgesamt höher ist (Giugni und Grasso 2021; Lilleker und Koc-Michalska 2017), diese Bereitschaft bei Klein(st)parteimitgliedern jedoch noch einmal deutlich stärker ausgeprägt ist.

Insgesamt lässt sich die Hypothese 4 nicht bestätigen, da Klein(st)parteimitglieder sich für ihre Mitgliedschaft keinen höheren Beitrag zum Funktionieren der Demokratie zuschreiben. Vielmehr nehmen alle Parteimitglieder ihre Mitgliedschaft und politische Partizipation als bedeutsam für Demokratie und Gesellschaft wahr.

Klein(st)parteimitglieder bekleiden in ihrer Partei häufiger Ämter als Mitglieder etablierter Parteien und schreiben sich entsprechend auch ein im Mittel höheres Aktivitätsniveau zu. In Anbetracht der ausgeprägten Aktivität von Klein(st)parteimitgliedern ist es überdies stimmig, dass sie das Eintreten von solchen Folgen der Parteimitgliedschaft, die im Rahmen des GIM den selektiven Anreizen zugeschrieben werden, insgesamt für realistischer halten (Tab. 7). Schließlich kommt derartiger Nutzen nur Mitgliedern zuteil, die an innerparteilichen Veranstaltungen partizipieren. Dabei gestaltet sich das Bild auf der Ebene der einzelnen Items allerdings etwas differenzierter. Hinsichtlich der selektiv, ergebnisbezogenen Anreize halten Klein(st)parteimitglieder nach ihrer persönlichen

13 Dabei scheinen sie sich im Mittel auch eher vorstellen zu können, Regeln zu brechen, wie zumindest die erhöhte Teilnahmebereitschaft an nichtgenehmigten Demonstrationen andeutet. 


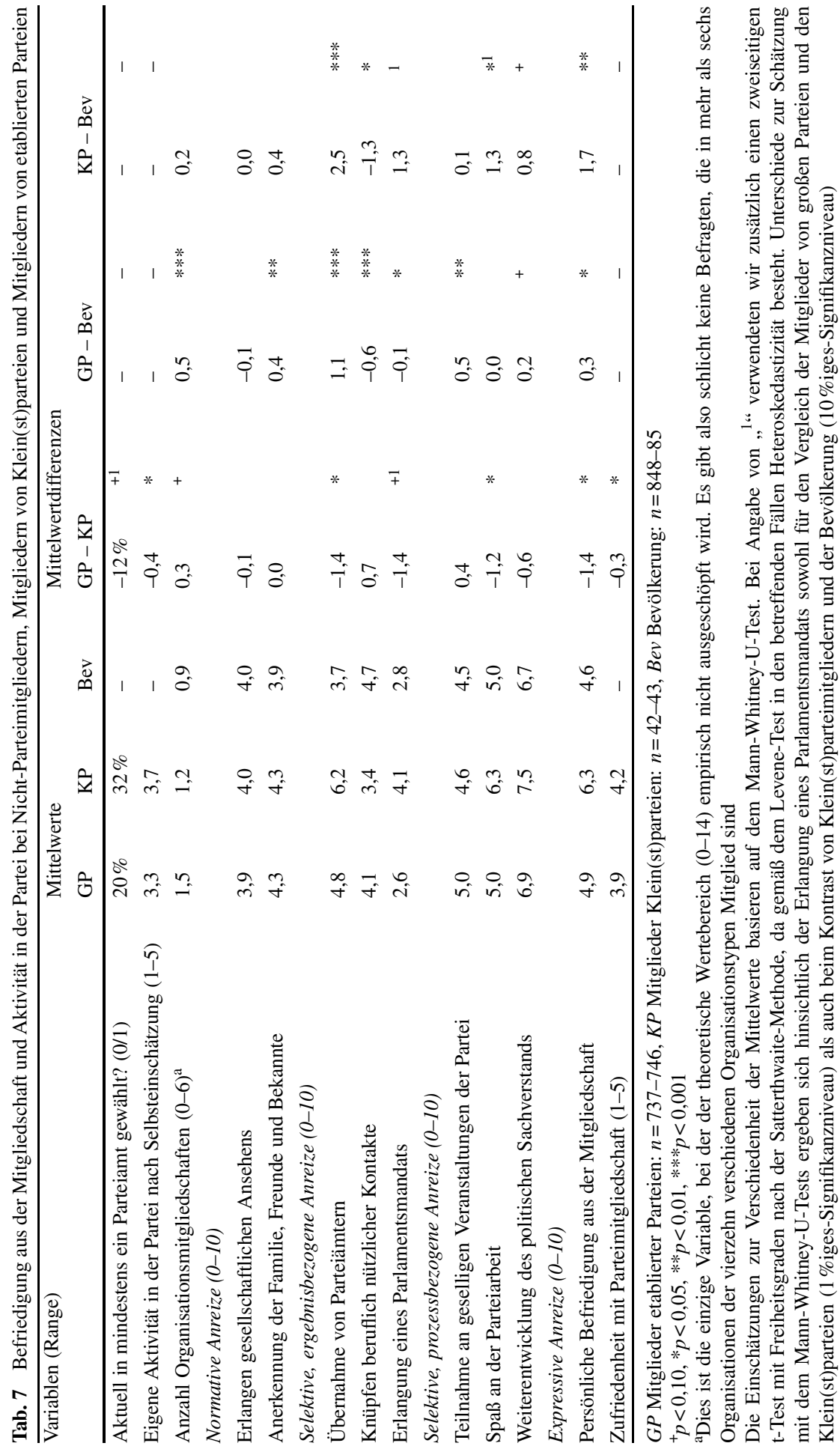


Erfahrung zwar die Erlangung von Parteiämtern und Mandaten für wahrscheinlicher als Mitglieder etablierter Parteien, glauben jedoch weniger an das Knüpfen wertvoller beruflicher Kontakte im Kontext der Parteiarbeit. Letzteres mag der quasi per definitionem niedrigeren Wahrscheinlichkeit geschuldet sein, Mitglieder der eigenen Klein(st)partei in beruflich relevanten Organisationen anzutreffen. Bei den selektiv, prozessbezogenen Anreizen erreicht lediglich eine Mittelwertdifferenz statische Signifikanz: Klein(st)parteimitglieder verbinden im Vergleich mit Mitgliedern etablierter Parteien mit ihrer Mitgliedschaft im Allgemeinen eher den Spaß an der Parteiarbeit. Die übrige Bevölkerung schließlich schreibt selektiven Anreizen, mit der deutlichen Ausnahme des Knüpfens beruflicher Kontakte, die geringste Relevanz zu. Für die normativen Anreize sind ferner keine nennenswerten Unterschiede zwischen den Gruppen beobachtbar. Es ist also nicht so, dass Mitglieder von Klein(st)parteien in höherem Maße Anerkennung von ihrem sozialen Umfeld aufgrund ihrer Tätigkeit erfahren. Auch scheinen Klein(st)parteimitglieder zwar stärker als die Gesamtbevölkerung am Vereinsleben teilzunehmen, jedoch verfügen Mitglieder etablierter Parteien entgegen unserer Erwartung nochmals über mehr Mitgliedschaften in anderen Organisationen. Hieraus lässt sich allerdings unter Umständen schließen, dass Klein(st)parteimitglieder ihre Mitgliedschaft als Ersatz für eventuelle Vereinstätigkeiten ansehen.

Abgeleitet aus den zugeschriebenen sozialen Beweggründen und einer erhöhten Aktivität von Klein(st)parteimitgliedern formulierten wir schließlich die Erwartung, dass Klein(st)parteimitglieder eine höhere Befriedigung aus ihrer Mitgliedschaft ziehen. Obgleich sich die Herleitung nur partiell als empirisch haltbar erweist - sind Klein(st)parteimitglieder doch nicht vor Ort stärker sozial vernetzt und erfahren keine höhere gesellschaftliche Anerkennung durch ihre Parteiarbeit - ergibt sich dennoch für Mitglieder kleiner Parteien recht deutlich, dass sie mit ihrer Mitgliedschaft zufriedener sind als Mitglieder von großen Parteien. Zudem zeigt sich anhand eines Items zur Erfassung des expressiven Anreizes, dass ihre aus der Mitgliedschaft erfahrene persönliche Befriedigung höher ist als jene von Mitgliedern etablierter Parteien, und auch höher als die von der Bevölkerung erwartete.

\section{Fazit}

Im Rahmen der Deutschen Parteimitgliederstudie 2009 kann festgestellt werden, dass die Mitglieder ,im Schatten der Macht“ sich deutlich aus den Kreisen der Unzufriedenen speisen. Es besteht eine Art U-Form der Bereitschaft einer Partei beizutreten. Menschen, die eher zufrieden oder sehr unzufrieden mit dem System sind, entscheiden sich für den Beitritt in eine Partei. Erstere Gruppe tritt eher einer großen Partei bei, letztere Gruppe eher einer Klein(st)partei. Dies bestätigten die Ergebnisse, die im Rahmen der Untersuchung der Mitglieder der Reformpartei in Kanada die Unzufriedenheit als starken Motivator aufzeigten (Clarke et al. 2000) und reiht sich ebenso in die Befunde zu neuen Parteien beziehungsweise ,third parties“ ein, die die Unzufriedenheit als erklärungsmächtigen Prädiktor der Wahlabsicht einer solchen Partei darlegten (Wuttke 2020; Allen und Brox 2005; Bélanger 2004). Dies erweitert die bisher einschlägigen Annahmen der Parteibeitrittsmoti- 
vation, die bei Parteimitgliedern hauptsächlich eine gewisse Zufriedenheit mit dem System herausstellten (Höhne 2018; Schäfer 2013; Anderson 1998), wenngleich dies nicht unbedingt auf die Mitglieder von Randparteien zutrifft (Höhne 2018).

Verbunden wird das Ganze bei den Klein(st)parteien mit einer starken ideologischen Motivation. Diese ist deutlich höher ausgeprägt als es bei den großen Parteien der Fall ist. Unsere Befunde relativieren die gängigen Annahmen der Motivationseigenschaften der Ideologie und insbesondere auch jene Ergebnisse zur Auswirkung radikalerer Ansichten (Whiteley et al. 2019). So ist Ideologie mitunter ein starker Motivator für die Parteimitgliedschaft an sich (Hoffmann und Springer 2019; Weber 2020), jedoch, verbunden mit einer extremeren ideologischen Ausprägung, ein begünstigender Faktor, sich in einer Klein(st)partei zu engagieren und nicht den Weg des Kompromisses in einer größeren Partei einzuschlagen. Dieser Effekt konnte auch bereits bei einer Untersuchung der ÖDP-Mitglieder festgestellt werden (Biehl und Kranenpohl 2011) und scheint somit ein wichtiger Faktor der Klein(st)parteimitgliedschaft zu sein. Dies heißt jedoch nicht, dass es nicht auch ideologische Abweichler in großen Parteien gibt (van Haute und Carty 2012). Nur stellen Klein(st)parteien eben auch eine Exit-Option für diese Mitglieder dar.

Grundsätzlich gibt es allerdings auch klare Überschneidungen zwischen Klein(st)parteimitgliedern und Mitgliedern von großen Parteien. Dies zeigt sich etwa bei den altruistischen Anreizen, also der Parteimitgliedschaft als Bürgerpflicht. Hier sind bei beiden Gruppen klare Differenzen zur allgemeinen Bevölkerung zu sehen, untereinander aber kaum. Gleichsam zeigt sich selbst bei Multi-Speed-Membership-Parteien, dass altruistische Anreize einen relativ großen Ausschlag dafür geben, ob man Mitglied wird oder nicht (Gomez et al. 2019). Wer also das Gefühl hat, die Mitgliedschaft sei in gewisser Weise eine Bürgerpflicht, engagiert sich auch eher in einer Partei - egal, ob Klein(st)- oder Großpartei.

An sich sind bei den selektiven Anreizen nicht sonderlich viele Unterschiede zu verzeichnen, außer dass Klein(st)parteimitglieder deutlich häufiger ein Amt bekleiden. Doch trotz des Mangels solcher Anreize zeigt sich, dass die Klein(st)parteimitglieder merklich zufriedener mit ihrer Partei sind als Mitglieder großer Parteien. Dies scheint zu bestätigen, dass es Klein(st)parteimitgliedern neben dem ideologischen- und Unzufriedenheitsaspekt eventuell auch um das soziale Miteinander in einer (kleinen) Gemeinschaft geht. Die Partei dient in diesem Falle also zum Teil als Ersatz für etwaige Vereinstätigkeiten. Zu bilanzieren ist somit, dass der Gang in den „Schatten der Macht“ vor allem durch Unzufriedenheit mit dem System, ideologisch radikale Einstellungen und durch den Spaß an der Parteiarbeit angetreten wird.

Die Ergebnisse deuten darauf hin, dass Mair und van Biezen (2001) durchaus zugestimmt werden kann, wenn Sie darauf verweisen, dass Parteien und andere traditionelle hierarchische Organisationen unter einer erhöhten Individualisierung der Gesellschaft leiden. Jedoch sollte die Aussage zunächst auf die großen Parteien beschränkt werden. Denn es scheint, als würde nicht das Konzept Partei an sich unter der erhöhten Individualisierung leiden, sondern hauptsächlich die großen Parteien. Die Individualisierung drückt sich also letztlich wahrscheinlich in einer höheren Anzahl von Klein(st)parteien aus, in denen, wie gezeigt, auch relativ viele Mitglieder zu finden sind. 
Für die Frage, warum sich Menschen für eine Parteimitgliedschaft entscheiden, reicht es nicht aus, nur die etablierten Parteien zu berücksichtigen. Dieser Beitrag hat versucht, die Motivationen der Klein(st)parteimitglieder aufzuzeigen. Dabei offenbarten sich durchaus große Unterschiede zwischen den Motivationen der Mitglieder großer und etablierter Parteien und denen der Mitglieder von Klein(st)parteien. Aufgrund der dürftigen Datenlage war es nur möglich, sich auf eine Befragung mit lediglich 31 Klein(st)parteimitgliedern zu stützen. Aus der geringen Fallzahl resultierte ein Fokus auf eben jene ausgeprägten Differenzen zwischen den untersuchten Gruppen. Auf Grundlage einer größeren Stichprobe ließe sich ein nuanciertes Bild zeichnen, zu dem dann auch multivariate Analysen beitrügen. Zudem stammen die Daten aus dem Jahr 2009. Gerade aufgrund der Tatsache, dass die Gruppe der Klein(st)parteien immer weiter zu wachsen scheint, wären neuere Daten begrüßenswert. Des Weiteren beschränkt sich diese Untersuchung auf Deutschland. Hier wären länderübergreifende Studien weiterhin gewinnbringend, gerade im Hinblick auch auf die unterschiedlichen Anreizstrukturen, die andere Wahlsysteme mit sich bringen. Beispielsweise in den Niederlanden, wo ein Einzug ins Parlament deutlich einfacher erscheint, könnten gewisse Ausprägungen (beispielsweise eine noch stärkere ideologische Motivation) verstärkt werden.

Schließlich ist eine Limitation detaillierter zu beschreiben: In der Parteimitgliederforschung gibt es nur ausgesprochen selten Panelbefragungen (für Großbritannien Whiteley und Seyd 2002; Seyd und Whiteley 2002). Dies erschwert es, bei der Ermittlung des Zusammenhangs von politischen Einstellungen und der Parteimitgliedschaft die Kausalrichtung zu bestimmen. In der vorliegenden Abhandlung besteht das Problem hauptsächlich für die Indikatoren politischer Unterstützung. Konkret stellt sich die Frage, ob Klein(st)parteien tatsächlich Unzufriedene anziehen oder nicht doch Unzufriedenheit gegenüber dem politischen System und seinen Akteuren vornehmlich erst durch den Kontakt mit anderen verdrossenen Mitgliedern entsteht. Diese Frage ist nicht mit letzter Gewissheit zu beantworten. Höhne (2018) stellt allerdings fest, dass das Vertrauen von Parteimitgliedern in die Demokratie in Deutschland und die Zufriedenheit mit dem Bundestag mit der Dauer der Mitgliedschaft zunimmt. Dies trifft für die systemkritischen Parteien Die Linke und die AfD gleichermaßen wie für die anderen parlamentarischen Parteien zu. Damit im Einklang beobachten wir, dass ein positiver Zusammenhang zwischen der Dauer der Mitgliedschaft in einer Klein(st)partei und der Demokratiezufriedenheit besteht $(\mathrm{r}=0,22)$. Diese Assoziation spricht gegen eine Radikalisierung in der Partei als Ursache für die geringe Zufriedenheit von Klein(st)parteimitgliedern.

Zusatzmaterial online Zusätzliche Informationen sind in der Online-Version dieses Artikels (https://doi. org/10.1007/s11615-021-00322-1) enthalten.

Funding Open Access funding enabled and organized by Projekt DEAL.

Open Access Dieser Artikel wird unter der Creative Commons Namensnennung 4.0 International Lizenz veröffentlicht, welche die Nutzung, Vervielfältigung, Bearbeitung, Verbreitung und Wiedergabe in jeglichem Medium und Format erlaubt, sofern Sie den/die ursprünglichen Autor(en) und die Quelle ordnungsgemäß nennen, einen Link zur Creative Commons Lizenz beifügen und angeben, ob Änderungen vorgenommen wurden. 
Die in diesem Artikel enthaltenen Bilder und sonstiges Drittmaterial unterliegen ebenfalls der genannten Creative Commons Lizenz, sofern sich aus der Abbildungslegende nichts anderes ergibt. Sofern das betreffende Material nicht unter der genannten Creative Commons Lizenz steht und die betreffende Handlung nicht nach gesetzlichen Vorschriften erlaubt ist, ist für die oben aufgeführten Weiterverwendungen des Materials die Einwilligung des jeweiligen Rechteinhabers einzuholen.

Weitere Details zur Lizenz entnehmen Sie bitte der Lizenzinformation auf http://creativecommons.org/ licenses/by/4.0/deed.de.

\section{Literatur und Weiterführende Literatur}

\section{Literatur}

Achury, Susan, Susan E. Scarrow, Karina Kosiara-Pedersen, und Emilie van Haute. 2020. The consequences of membership incentives: do greater political benefits attract different kinds of members? Party Politics 26(1):56-68. https://doi.org/10.1177/1354068818754603.

Allen, Neal, und Brian J. Brox. 2005. The roots of third party voting. Party Politics 11(5):623-637. https:// doi.org/10.1177/1354068805054983.

Amorim Neto, Octavio, und Gary W. Cox. 1997. Electoral institutions, cleavage structures and the number of parties. American Journal of Political Science 41(1):149-174.

Anderson, Christopher J. 1998. Parties, party systems, and satisfaction with democratic performance in the new Europe. Political Studies 46(3):572-588.

Behnke, Joachim. 2007. Das Wahlsystem der Bundesrepublik Deutschland. Logik, Technik und Praxis der Verhältniswahl, 1. Aufl., Baden-Baden: Nomos.

Bélanger, Éric. 2004. Antipartyism and third-party vote choice. Comparative Political Studies 37(9): 1054-1078. https://doi.org/10.1177/0010414004268847.

Biehl, Heiko, und Uwe Kranenpohl. 2011. Große Politik in einer kleinen Partei. Strukturen und Determinanten innerparteilicher Partizipation in der Ökologisch-Demokratischen Partei (ödp). Mitteilungen des Instituts für Deutsches und Internationales Parteienrecht und Parteienforschung 17:93-110.

Bolleyer, Nicole. 2013. New parties in old party systems. Persistence and decline in seventeen democracies, 1. Aufl., Comparative politics. Oxford: Oxford University Press.

van den Boom, Dirk. 1999. Politik diesseits der Macht? Zu Einfluß, Funktion und Stellung von Kleinparteien im politischen System der Bundesrepublik Deutschland. Wiesbaden: VS.

Borisyuk, Galina, Colin Rallings, Michael Thrasher, und Henk van der Kolk. 2007. Voter support for minor parties. Party Politics 13(6):669-693. https://doi.org/10.1177/1354068807081816.

Chamberlain, Adam. 2012. The growth of third-party voting. State Politics \& Policy Quarterly 12(3): 343-361. https://doi.org/10.1177/1532440012451155.

Clarke, Harold D., Allan Kornberg, Ellis Faron, und Jon Rapkin. 2000. Not for fame or fortune. Party Politics 6(1):75-93.

Dalton, Russell J. 2000. Citizen attitudes and political behaviour. Comparative Political Studies 33(6/7): 912-940.

Ferejohn, John A., und Morris P. Fiorina. 1974. The paradox of not voting: a decision theoretic analysis. American Political Science Review 68(2):525-536.

Giugni, Marco, und Maria Grasso. 2021. Party membership and social movement activism: a macro-micro analysis. Party Politics 27(1):13:92-102. https://doi.org/10.1177/1354068818823446.

Gomez, Raul, Luis Ramiro, Laura Morales, und Jaime Aja. 2019. Joining the party: Incentives and motivations of members and registered sympathizers in contemporary multi-speed membership parties. Party Politics. https://doi.org/10.1177/1354068819891047.

Hartmann, Jürgen. 2013. Das politische System der BRD im Kontext. Wiesbaden: Springer.

van Haute, Emilie, und R. Kenneth Carty. 2012. Ideological misfits: a distinctive class of party members. Party Politics 18(6):885-895. https://doi.org/10.1177/1354068810395058.

Heidar, Knut. 1994. The polymorphic nature of party membership. European Journal of Political Research 25(1):61-86. https://doi.org/10.1111/j.1475-6765.1994.tb01201.x.

Hirano, Shigeo, und James M. Snyder. 2007. The decline of third-party voting in the United States. The Journal of Politics 69(1):1-16. https://doi.org/10.1111/j.1468-2508.2007.00490.x.

Hirschman, Albert. 1970. Exit, voice, and loyalty. Responses to decline in firms, organizations, and states. Cambridge: Harvard University Press. 
Hoffmann, Hanna. 2011. Warum werden Bürger Mitglied in einer Partei? In Parteimitglieder in Deutschland, 1. Aufl., Hrsg. Tim Spier, Markus Klein, Ulrich von Alemann, Hanna Hoffmann, Annika Laux, Alexandra Nonnenmacher, und Katharina Rohrbach, 79-95. Wiesbaden: VS.

Hoffmann, Hanna, und Frederik Springer. 2019. The individual-level determinants of German party membership. German Politics 28(2):242-261. https://doi.org/10.1080/09644008.2019.1603295.

Höhne, Benjamin. 2018. Engagement beugt Politikverdrossenheit (nicht immer) vor. Demokratievertrauen und Parlamentszufriedenheit von aktiven Parteimitgliedern. Zeitschrift für Parlamentsfragen 49(4):919-932. https://doi.org/10.5771/0340-1758-2018-4-919.

Hooghe, Marc, und Ann-Kristin Kölln. 2020. Types of party affiliation and the multi-speed party. Party Politics 26(4):355-365. https://doi.org/10.1177/1354068818794220.

Jesse, Eckhard. 2018a. Deutsche Kommunistische Partei (DKP). In Handbuch der deutschen Parteien, 3. Aufl., Hrsg. Frank Decker, Viola Neu, 283-285. Wiesbaden: Springer VS.

Jesse, Eckhard. 2018b. Nationaldemokratische Partei Deutschlands (NPD). In Handbuch der deutschen Parteien, 3. Aufl., Hrsg. Frank Decker, Viola Neu, 407-417. Wiesbaden: Springer VS.

Köhler, Jan. 2006. Nicht-etablierte Parteien: Funktionen und Rechtsfragen. In Kleine Parteien im Aufwind: Zur Veränderung der deutschen Parteienlandschaft, Hrsg. Uwe Jun, Henry Kreikenbom, und Viola Neu, 39-57. New York, Frankfurt am Main: Campus.

Koo, Sejin. 2021. Does policy motivation drive party activism? A study of party activists in three Asian democracies. Party Politics 27(1):187-201. https://doi.org/10.1177/1354068820908021.

Kranenpohl, Uwe. 2008. Die ,christlichen Grünen“. Sozialstruktur und politische Einstellungen der Mitglieder der Ökologisch-Demokratischen Partei (ödp). Historisch-Politische Mitteilungen 15(1):47-62. https://doi.org/10.7788/hpm.2008.15.1.47.

Kranenpohl, Uwe. 2018. Ökologisch-Demokratische Partei (ÖDP). In Handbuch der deutschen Parteien, 3. Aufl., Hrsg. Frank Decker, Viola Neu, 425-431. Wiesbaden: Springer VS.

Kranenpohl, Uwe, und Oskar Niedermayer. 2013. Kleinstparteien. In Handbuch Parteienforschung, Hrsg. Oskar Niedermayer, 663-681. Wiesbaden: Springer.

Kselman, Daniel M., Eleanor Neff Powell, und Joshua A. Tucker. 2016. Crowded space, fertile ground: party entry and the effective number of parties. Political Science Research and Methods 4(2):317-342. https://doi.org/10.1017/psrm.2015.22.

Küppers, Anne. 2018. Partei für Gesundheitsforschung (Gesundheitsforschung). In Handbuch der deutschen Parteien, 3. Aufl., Hrsg. Frank Decker, Viola Neu, 440-441. Wiesbaden: Springer VS.

Laux, Annika. 2011. Was motiviert Parteimitglieder zum Beitritt? In Parteimitglieder in Deutschland, 1. Aufl., Hrsg. Tim Spier, Markus Klein, Ulrich von Alemann, Hanna Hoffmann, Annika Laux, Alexandra Nonnenmacher, und Katharina Rohrbach, 61-78. Wiesbaden: VS.

Lee, Daniel J. 2014. Third-party threat and the dimensionality of major-party roll call voting. Public Choice 159(3-4):515-531. https://doi.org/10.1007/s11127-013-0066-X.

Lewandowsky, Marcel. 2018. Die Violetten - für spirituelle Politik (Die Violetten). In Handbuch der deutschen Parteien, 3. Aufl., Hrsg. Frank Decker, Viola Neu, 515-516. Wiesbaden: Springer VS.

Lilleker, Darren G., und Karolina Koc-Michalska. 2017. What drives political participation? Motivations and mobilization in a digital age. Political Communication 34(1):21-43. https://doi.org/10.1080/ 10584609.2016.1225235.

Luks, Samantha, Joanne M. Miller, und Lawrence R. Jacobs. 2003. Who wins? Campaigns and the third party vote. Presidential Studies Quarterly 33(1):9-30. https://doi.org/10.1177/0360491802250540.

Magin, Raphael, Markus Freitag, und Adrian Vatter. 2009. Cleavage structures and voter alignments within nations. Zeitschrift für Vergleichende Politikwissenschaft 3(2):231-256. https://doi.org/10.1007/ s12286-009-0062-1.

Mair, Peter, und Ingrid van Biezen. 2001. Party membership in twenty European democracies, 1980-2000. Party Politics 7(1):5-21.

Meguid, Bonnie M. 2005. Competition between unequals: the role of mainstream party strategy in niche party success. American Political Science Review 99(3):347-359.

Meyer, Thomas M., und Bernhard Miller. 2015. The niche party concept and its measurement. Party Politics 21(2):259-271. https://doi.org/10.1177/1354068812472582.

Müller, Frank, und Rainer Traub. 2004. Motivstrukturen der Stuttgarter Parteimitglieder. In Städte und Regionen in Europa Parteien, Parteieliten und Mitglieder in einer Großstadt, Bd. 11, Hrsg. Melanie Walter-Rogg, Oscar W. Gabriel, 25-47. Wiesbaden: VS.

Nestler, Christian. 2013. Die Kleinstparteien in Mecklenburg-Vorpommern. In Politik in Mecklenburg-Vorpommern, Hrsg. Martin Koschkar, Christian Nestler, und Christopher Scheele, 169-185. Wiesbaden: Springer. 
Niedermayer, Oskar. 2020. Parteimitglieder in Deutschland: Version 2020. Arbeitshefte aus dem OttoStammer-Zentrum 31.

Poletti, Monica, Paul Webb, und Tim Bale. 2019. Why do only some people who support parties actually join them? Evidence from Britain. West European Politics 42(1):156-172. https://doi.org/10.1080/ 01402382.2018.1479921.

Raymond, Christopher D., und Mathias Wessel Tromborg. 2016. What's information got to do with it? Third-party voting in plurality systems. Party Politics 22(4):534-543. https://doi.org/10.1177/ 1354068814551295.

Rohrbach, Katharina. 2013. Partizipation in politischen Parteien. Eine empirische Analyse des Parteibeitritts, der Aktivität und des Parteiaustritts, 1. Aufl., Frankfurt a.M: Peter Lang.

Rowold, Manfred. 1974. Im Schatten der Macht. Zur Oppositionsrolle der nicht-etablierten Parteien in der Bundesrepublik. Bonner Schriften zur Politik und Zeitgeschichte, Bd. 9. Düsseldorf: Droste.

Sartori, Giovanni. 1976. Parties and party systems. A framework for analysis. Cambridge: Cambridge University Press.

Schäfer, Armin. 2013. Affluence, inequality and satisfaction with democracy. In Society and democracy in Europe Routledge advances in European politics, Bd. 89, Hrsg. Silke I. Keil, Oscar W. Gabriel, 139-161. London: Routledge.

Schulze, Andreas. 2018. Die Grauen - Graue Panther (GRAUE). In Handbuch der deutschen Parteien, 3. Aufl., Hrsg. Frank Decker, Viola Neu, 363-368. Wiesbaden: Springer VS.

Seyd, Patrick, und Paul F. Whiteley. 2002. New Labour's grassroots. The transformation of the Labour Party membership, 1. Aufl., Basingstoke: Palgrave Macmillan.

Seyd, Patrick, und Paul F. Whiteley. 1992. Labour's grass roots. The politics of party membership. Oxford: Clarendon Press.

Speit, Andreas. 2020. Nicht verbotene rechte Gruppen: Ein deutsches Gruselkabinett. taz. https://taz.de/ Nicht-verbotene-rechte-Gruppen/!5656138/. Zugegriffen: 1. März 2021.

Spier, Tim. 2019. Not dead yet? Explaining party member activity in Germany. German Politics 28(2):282-303. https://doi.org/10.1080/09644008.2018.1528237.

Spier, Tim, Markus Klein, Ulrich von Alemann, Hanna Hoffmann, Annika Laux, Alexandra Nonnenmacher, und Katharina Rohrbach (Hrsg.). 2011. Parteimitglieder in Deutschland, 1. Aufl., Wiesbaden: VS.

Vetter, Angelika. 2004. Die idealen Staatsbürger? Politische Involvierung und Unterstützung von Parteimitgliedern. In Parteien, Parteieliten und Mitglieder in einer Großstadt Städte und Regionen in Europa, Bd. 11, Hrsg. Melanie Walter-Rogg, Oscar W. Gabriel, 125-148. Wiesbaden: VS.

Weber, Regina. 2020. Why do young people join parties? The influence of individual resources on motivation. Party Politics 26(4):496-509. https://doi.org/10.1177/1354068818792576.

Wehling, Hans-Georg. 2007. Freie Wähler (FW/FWG). In Handbuch der deutschen Parteien Schriftenreihe der Bundeszentrale für politische Bildung, Bd. 640, Hrsg. Frank Decker, 288-294. Bonn: Bundeszentrale für politische Bildung.

Weldon, Steven. 2006. Downsize My polity? The impact of size on party membership and member activism. Party Politics 12(4):467-481.

Whiteley, Paul F., und Patrick Seyd. 2002. High-intensity participation. The dynamics of party activism in Britain. Ann Arbor: Michigan University Press.

Whiteley, Paul F., Erik Larsen, Matthew Goodwin, und Harold Clarke. 2019. Party activism in the populist radical right: the case of the UK independence party. Party Politics. https://doi.org/10.1177/ 1354068819880142.

Wuttke, Alexander. 2020. New political parties through the voters' eyes. West European Politics 43(1):22-48. https://doi.org/10.1080/01402382.2019.1603940.

Zimmer, Almut. 2017. Magdeburger Gartenpartei. https://www.bpb.de/politik/wahlen/wer-steht-zur-wahl/ bundestagswahl-2017/254653/mg. Zugegriffen: 01. März 2021.

\section{Weiterführende Literatur}

Gerring, John. 2005. Minor parties in plurality electoral systems. Party Politics 11(1):79-107. https://doi. org/10.1177/1354068805048474. 
Jan-Eric Bartels ist wissenschaftlicher Mitarbeiter am Lehrstuhl für das politische System der Bundesrepublik Deutschland des Instituts für Politikwissenschaft an der Georg-August-Universität Göttingen. Seine Forschungsgebiete umfassen unter anderem Parteienwettbewerb und Kleinstparteien.

Frederik Springer ist wissenschaftlicher Mitarbeiter am Institut für Politikwissenschaft der Leibniz Universität Hannover. Seine Forschungsgebiete umfassen die Wahl \& Wahlsystemforschung sowie die Parteienforschung. 